\title{
MOLECULES IN G1.6-0.025 - "HOT" CHEMISTRY IN THE ABSENCE OF STAR FORMATION AT THE PERIPHERY OF THE GALACTIC CENTER REGION
}

\author{
Karl M. Menten ${ }^{1}$, Robert W. Wilson ${ }^{2}$, Silvia Leurini ${ }^{3}$, and Peter Schilke ${ }^{1}$
}

Received —

\footnotetext{
${ }^{1}$ Max-Planck-Institut für Radioastronomie, Auf dem Hügel, D-53121 Bonn, Germany kmenten, pschilke@mpifr-bonn.mpg.de

${ }^{2}$ Harvard-Smithsonian Center for Astrophysics, 60 Garden Street/MS42, Cambridge MA 02138

rwilson@cfa.harvard.edu

${ }^{3}$ European Southern Observatory, Karl-Schwarzschild-Strasse 2, D-85748 Garching sleurini@eso.org
} 


\begin{abstract}
We present molecular line mapping of the Giant Molecular Cloud G1.6-0.025, which is located at the high longitude end of the Central Molecular Zone of our Galaxy. We assess the degree of star formation activity in that region using several tracers and find very little. We made a large scale, medium $\left(2^{\prime}\right)$ resolution map in the $J=2-1$ transition of $\mathrm{SiO}$ for which we find clumpy emission over $\mathrm{a} \sim 0.8 \times 0.3$-sized region stretching along the Galactic plane. Toward selected positions we also took spectra in the easy to excite $J_{k}=2_{k}-1_{k}$ quartet of $\mathrm{CH}_{3} \mathrm{OH}$ and the CS $2-1$ line. Throughout the cloud these $\mathrm{CH}_{3} \mathrm{OH}$ lines are, remarkably, several times stronger than, both, the $\mathrm{CS}$ and the $\mathrm{SiO}$ lines. The large widths of all the observed lines, similar to values generally found in the Galactic center, indicate a high degree of turbulence. Several high LSR velocity clumps that have 50-80 $\mathrm{km} \mathrm{s}^{-1}$ higher velocities than the bulk of the molecular cloud appear at the same projected position as "normal" velocity material; this may indicate cloudcloud collisions. Statistical equilibrium modeling of the $\mathrm{CH}_{3} \mathrm{OH}$ lines observed by us and others yield relatively high densities and moderate temperatures for a representative dual velocity position. We find $810^{4} \mathrm{~cm}^{-3} / 30 \mathrm{~K}$ for material in the G1.6-0.025 cloud and a higher temperature (190 K), but a 50\% lower density in a high velocity clump projected on the same location. Several scenarios are discussed in which shock chemistry might enhance the $\mathrm{CH}_{3} \mathrm{OH}$ and $\mathrm{SiO}$ abundances in G1.6-0.025 and elsewhere in the Central Molecular Zone.
\end{abstract}

Subject headings: ISM: clouds — ISM: molecules — Galaxy: center 


\section{INTRODUCTION - THE CENTRAL MOLECULAR ZONE AND ITS (LITTLE) STAR FORMATION ACTIVITY}

"Ordinary" giant molecular clouds (GMCs) in the Galactic disk have sizes of tens of parsecs, temperatures, $T_{\text {kin }}$, in the low tens of $\mathrm{K}$, and densities, $n$, of order a hundred $\mathrm{cm}^{-3}$. Embedded in these GMCs are regions of star formation with much higher temperatures and densities. In contrast, the gas in the central molecular zone (CMZ; see e.g. Morris \& Serabyn 1996) of our Galaxy, stretching from Galactic latitude,l, $\sim+1.6$ to -1.1 in $\mathrm{a} \sim \pm 0.3$ wide band in latitude, $b$, around the Galactic center (GC, Bally et al. 1987, 1988; Dahmen et al. 1997, 1998; Oka et al. 1998) is in general characterized by much higher temperatures, densities, and more turbulence, resulting in larger linewidths ( $\gtrsim 10-30$ $\left.\mathrm{km} \mathrm{s}^{-1}\right)$. Güsten \& Philipp (2004) give a recent review.

To get a handle on the chemistry and physical conditions in these peculiar clouds, multi-transition measurements of molecules other than the ubiquitous and easily-thermalized carbon monoxide (CO) are highly desirable. By observing many lines from a given species and modeling the results using, e.g., large velocity gradient (LVG) methods, one can derive the densities and temperatures of the GC clouds, which are known to be significantly higher than values in Galactic disk clouds, but still relatively ill-constrained by existing data. Extensive surveys of carbon monosulfide (CS), ${ }^{13} \mathrm{CO}$, and $\mathrm{C}^{18} \mathrm{O}$ have been made mostly with coarse spatial resolutions of $2^{\prime}$ and $9^{\prime}$, respectively (Bally et al. 1987; Dahmen et al. 1997) and, in a smaller region around the $\mathrm{GC}$ itself, of cyanoacetylene $\left(\mathrm{HC}_{3} \mathrm{~N}\right)$, ammonia $\left(\mathrm{NH}_{3}\right)$, and silicon monoxide $(\mathrm{SiO})$ with beam sizes between $40^{\prime \prime}$ and $140^{\prime \prime}$ (Güsten et al. 1981; Walmsley et al. 1986; Martin-Pintado et al. 1997). Walmsley et al. observed several $\mathrm{HC}_{3} \mathrm{~N}$ transitions within $1^{\prime} .5$ of the $\mathrm{GC}$ and found that the bulk of the gas has $T \approx 80 \mathrm{~K}$ and $n \approx 10^{4} \mathrm{~cm}^{-3}$, while $20 \%$ of its mass may exist in higher $\left(10^{5} \mathrm{~cm}^{-3}\right)$ density clumps. Astonishingly, a large number of complex organic molecules have been found to show 
widespread emission all over the CMZ (see $96 \underline{6}$ ).

Whether tidal forces, stronger turbulence, higher densities, and stronger magnetic fields (compared to Galactic disk clouds) favor stars formation or hinder it can be argued either way. Fact is that the spectacular Arches and Quintuplet clusters give testimony for violent star formation a few million years ago (Figer et al. 1999, 2002). Figer et al. (2004) argue that ongoing star formation is responsible for the observed central stellar cusp. From Infrared Space Observatory (ISO) and Spitzer Space Telescope infrared wavelength data Schuller et al. (2005) derive a star formation rate in the CMZ of $0.2 M_{\odot} \mathrm{yr}^{-1}$ over the past $\sim 0.5$ Myr, which is an appreciable fraction of our whole Galaxy's star formation rate.

However, apart from the star formation-wise extremely active Sgr B2 region, little of what is commonly assumed to be evidence for on-going high-mass star formation is found in the GC GMCs at present, such as (ultra)compact $\mathrm{HII}$ regions, $\mathrm{H}_{2} \mathrm{O}$ and $\mathrm{CH}_{3} \mathrm{OH}$ masers. The submillimeter-detected dust ridge identified by Lis \& Carlstrom (1994) marks highest column density material of the general GC dust emission/molecular cloud distribution (Pierce-Price et al. 2000). It has the potential to harbor proto-cluster cores with the massive $\left(1 \quad 10^{6} M_{\odot}\right)$ Infrared Dark Cloud M0.25+0.11 the most prominent example (Lis et al. 1994; Lis \& Menten 1998). However, in this ridge, namely in M0.25+0.11, and also in the larger GC region only few markers of star formation have been found, just very few $\mathrm{H}_{2} \mathrm{O}$ masers and compact continuum sources (Güsten \& Downes 1981; Lis et al. 1994). Caswell (1996) covered an area of extent $l= \pm 0 .^{\prime \prime} 9, b= \pm 0 .^{\prime \prime} 5$ in a sensitive interferometric search for 6.7 GHz class II methanol masers, which are unambiguous tracers of high-mass star formation. He only found 23 masers sites with half of them located in the Sgr B2 complex alone. 


\section{THE PECULIAR G1.6-0.025 MOLECULAR CLOUD}

\subsection{Extended, Warm, Molecular Gas}

The molecular cloud G1.6-0.0251 lies at the very easternmost longitude edge of the cloud complexes making up the $\mathrm{CMZ}$ that were mapped in $\mathrm{CO},{ }^{13} \mathrm{CO}, \mathrm{C}^{18} \mathrm{O} \mathrm{CS}$, and other molecules (Bally et al. 1987, 1988; Dahmen et al. 1997, 1998; Oka et al. 1998; Martin et al. 2004) Maybe because of its location, this cloud received comparatively little attention in the past. However, the few observations that do exist reveal a fascinating chemical picture.

For further reference, we show, in Fig. 1, an image of the velocity-integrated emission in the $J=2-1$ transition of $\mathrm{SiO}$ discussed in 4.1 ,

Apart from the low resolution surveys in CS and $\mathrm{C}^{18} \mathrm{O}$ G1.6-0.025 has been mapped in several inversion transitions of $\mathrm{NH}_{3}$ by Gardner et al. (1985), who found remarkably strong emission in the $(J, K)=(1,1),(2,2)$, and $(3,3)$ lines, with the $(3,3)$ line possibly being inverted toward one position. The $\mathrm{NH}_{3}$ observations indicate that the cloud is warm (probably $>50 \mathrm{~K}$ ) but Gardner et al. do not give a thorough discussion of the temperatures suggested by the $\mathrm{NH}_{3}$ data. Gardner \& Boes (1987), based on another set of $\mathrm{NH}_{3}$ data, conclude $T>120 \mathrm{~K}$. Kuiper et al. (1993) observed the $1_{10}-1_{01}$ transition of $\mathrm{C}_{3} \mathrm{H}_{2}$ near 18.3 GHz. This line, whose appearance is ubiquitous in molecular clouds, here appears atypically in enhanced absorption against the cosmic microwave background radiation.

Also overcooling was found by Whiteoak \& Peng (1989) in the $2_{0}-3_{-1} E$ line of

\footnotetext{
${ }^{1}$ Strictly speaking G1.6-0.025 is only the cloud fragment delineated by the square in Fig. 1. For simplicity's sake, we refer by that name to the whole $\sim 0: 8 \times 00^{\circ} 35$-sized region shown in that figure.
} 
$\mathrm{CH}_{3} \mathrm{OH}$. This transition, which is the second strongest class II methanol maser line 2 (Batrla et al. 1987), has been found in absorption in dark clouds (Walmsley et al. 1988), which lack the far-infrared continuum emission necessary to produce class II maser action and also in absorption in class I methanol maser regions for which the same is true. Whiteoak \& Peng (1989) model the very (up to $2 \mathrm{~K}$ ) deep absorption and find a high E-type $\mathrm{CH}_{3} \mathrm{OH}$ abundance of $10^{-7}$ and an $\mathrm{H}_{2}$ density $\sim 10^{4} \mathrm{~cm}^{-3}$. Haschick \& Baan (1993) find extended, strong maser emission in the (class I) methanol maser $4_{-1}-3_{0} E$ line. As explained in $\$ 5.1$, the occurrence of overcooling in the $2_{0}-3_{-1} E$ and simultaneous maser action in the $4_{-1}-3_{0} E$ lines is expected from basic properties of the methanol molecule and can be used to constrain the physical conditions in the masing region.

Sobolev (1992) and Salii et al. (2002) discuss observations of several lines from methanol and other molecules with the RT-22 telescope at Pushchino (with 2' resolution) and the Swedish-ESO Submillimeter Telescope (SEST) (with $\approx 0.5$ and $\approx 1^{\prime}$ resolution), which they model to derive physical parameters. Their results are compared to our own in $\$ 5.1$.

One of the most astonishing things about G1.6-0.025 is the virtually complete absence of any marker of high-mass star formation on which we shall elaborate in $\$ 2.2$. This means, in G1.6-0.025 we have the opportunity to study large-scale high-temperature gas-phase chemistry in the absence of strong UV fields, which, in star-forming regions occurs only in very high extinction, spatially compact hot cores near young (proto)stars. In the latter environment, the high observed abundances of $\mathrm{SiO}, \mathrm{CH}_{3} \mathrm{OH}$, and other more complex species, which are orders of magnitudes or more higher than their quiescent, cool molecular cloud abundances, are thought to result from the evaporation of ice grain mantles in which these molecules resided in frozen-out form (see, e.g. Garrod \& Herbst 2006, and references

\footnotetext{
${ }^{2}$ See Menten (1991) for the nomenclature/classification of $\mathrm{CH}_{3} \mathrm{OH}$ masers.
} 
therein). Later in this paper (in \$5.2) we will argue that in the case of G1.6-0.025 these abundances may be the result of shock chemistry.

Given that several of the cloud's characteristics (high linewidths, high abundances of usually rare species) are typical for GC GMCs, we assume in the following that is at the distance of the GC, $8 \mathrm{kpc}$ (Reid 1993) . Other evidence for placing the cloud there are the high $\left[\mathrm{H}_{2}^{13} \mathrm{CO}\right] /\left[\mathrm{H}_{2} \mathrm{CO}\right]$ and $\left[\mathrm{H}_{2}^{13} \mathrm{CO}\right] /\left[\mathrm{H}_{2} \mathrm{C}^{18} \mathrm{O}\right]$ isotopic abundance ratios, which are by factors of 4 and 2 higher, respectively, than values found in the solar neighborhood, but typical for the GC region (Gardner \& Whiteoak 1981, 1982). Its projected distance from the $\mathrm{GC}$ is ca. 200 pc.

In this paper we report medium spatial resolution $\left(2^{\prime}\right)$ mapping observations of G1.6-0.025 in the $J=2-1$ rotational line of silicon monoxide ( $\mathrm{SiO}$ ). Furthermore, we observed selected positions in the $2-1$ line of carbon monosulfide (CS) and several methanol $\left(\mathrm{CH}_{3} \mathrm{OH}\right)$ transitions. As described by Leurini et al. (2004), newly calculated collisional rate coefficients now allow meaningful modeling of methanol excitation to obtain densities and kinetic temperatures (see \$5.1). Using all this information, we summarize the available chemical information for G1.6-0.025 and in $\$ 5.2$ discuss possible reasons for the observed picture.

\section{2. (Almost Non-)Existent Active Massive Star Formation in G1.6-0.025}

In order to assess whether G1.6-0.025's peculiar chemistry could be energetically driven by the influence of young high-mass stars, we conducted a census of observational phenomena and, in particular, of tracers of on-going high-mass star formation activity in the region and its surroundings. For this, we conducted a literature search using the 
SIMBAD 3 database. We searched for all astronomical objects contained in that database in a circle of 0.3 radius with $l, b=1.4,0 \circ 0$ at its center. We found a total of 138 objects, many of which are foreground (some of them $\mathrm{OH} / \mathrm{IR}$ ) stars and planetary nebulae. However, also a few compact radio continuum sources were found, for some of which, as discussed in the following, multi-wavelength data are available, allowing a characterization.

For our purposes, we are only interested in objects that are (most probably) associated with G1.6-0.025 and may have some influence on their environment. Given this, it is straightforward to eliminate radio sources from our list that are not coincident with IRAS point sources. A spot check reveals that, e.g., G1.285-0.054, which has no associated IRAS source is, both, variable and has a negative, non-thermal spectral index and is therefore in all likelihood of extragalactic origin. On the other hand, IRAS sources without detected radio emission may be important, as there is no established correlation between the radio and IR luminosities of very young high-mass protostellar objects; see the famous case of Orion IRc2 (Menten \& Reid 1995).

$\mathrm{OH} / \mathrm{IR}$ objects are easy to eliminate from our list since they can be identified by their IRAS colors (as established by van der Veen \& Habing 1988) and/or the presence of 1612 $\mathrm{MHz} \mathrm{OH}$ maser emission.

The only IRAS sources in the general region that are definitely associated with star formation are IRAS 17450-2742 and 17457-2743 which are coincident with the compact HII regions Sgr D 7 and 8, respectively (Liszt 1992); the latter one is also known as GPSR5 1.396-0.006 (Becker et al. 1994). We note that these sources are not coincident with any molecular peak in the cloud. Observed and derived properties for them can be found in Table 1. While Liszt determines for Sgr D 7 a size of $1^{\prime}$ at $1.6 \mathrm{GHz}$, he finds source 8

\footnotetext{
${ }^{3}$ http://simbad.u-strasbg.fr/Simbad
} 
unresolved in his $13^{\prime \prime} \times 23^{\prime \prime}$ beam. Becker et al. (2004), with $4^{\prime \prime}$ beam size, do not detect 7 at either 1.4 or $5 \mathrm{GHz}$, most certainly "resolving it out" and find source 8 unresolved at either frequency with flux densities of 24.2 and 26 mJy at 1.4 and $5 \mathrm{GHz}$, respectively. Assuming a size of $2^{\prime \prime}$ for source 8 and an electron temperature of $10000 \mathrm{~K}$ we calculate an optical depth, $\tau$, of the free-free emission of $0.47,0.29$, and 0.03 at 1.4, 1.6, and $5 \mathrm{GHz}$ respectively. For source 7 we determine $\tau=0.003$. Using the formula given by Mezger et al. (1974) we derive that Lyman continuum photon fluxes of $1.1 \times 10^{48} \mathrm{~s}^{-1}$ and $1.4 \times 10^{47} \mathrm{~s}^{-1}$ are needed to produce the compact HII regions Sgr D 7 and 8, respectively. According to the Tables given by Panagia (1973) these values correspond to ZAMS spectral types of O9 and $\mathrm{B} 0$, respectively.

To determine the neutral gas masses and luminosities of these sources from the IRAS data in the same way as described by Lis \& Menten (1998), Planck functions were fitted to the measured 12, 25, 60, and $100 \mu \mathrm{m}$ flux densities 4 to determine dust temperatures (see Table 11). We used the formulae given therein and in the paper by Motte et al. (2003) to determine the gas masses listed in Table 1 for Sgr D 7 and 8 (taking the upper limits in the table at face value). Integrating over the spectral energy distributions we derive the bolometric luminosities given in Table 1, which, again according to Panagia (1973), are produced by a $\mathrm{B} 0$ and a B1 ZAMS star, respectively. These spectral types are very similar to the ones inferred from the Lyman continuum fluxes.

All in all we conclude that, apart from the two sources discussed above, there is presently very little star-forming activity in G1.6-0.025 and consider it highly unlikely that star-formation activity contributes to its enhanced temperature in a significant way.

\footnotetext{
${ }^{4}$ This fitting was not straightforward as the 12,60 , and $100 \mu \mathrm{m}$ flux densities listed by the SIMBAD database for Sgr D 7 are very uncertain, as is the $60 \mu \mathrm{m}$ value for D 8; for the latter source only upper limits are given for the 12 and $100 \mu \mathrm{m}$ flux densities.
} 


\subsection{A Possible Connection to the G1.4-0.1 Supernova Remnant}

The supernova remnant (SNR) G1.4-0.1, which lies partially in the area mapped by us clearly interacts with molecular gas. Yusef-Zadeh et al. (1999) found $1720 \mathrm{MHz} \mathrm{OH}$ maser emission at $l, b=1.4164,-0^{\circ} 1323$ (see Fig. 1) at a velocity of $-2.4 \mathrm{~km} \mathrm{~s}^{-1}$, which is blue-shifted relative to almost all of the gas we observe. Possibly the masing gas is located in the portion of the swept-up molecular material coming toward us and thus amplifying the

continuum background. Lockett et al. (1999) constrain the conditions for the occurrence of these masers to temperatures between 50 and $125 \mathrm{~K}$, densities and $\mathrm{OH}$ column densities around $10^{5} \mathrm{~cm}^{-3}$ and $10^{16} \mathrm{~cm}^{-2}$, respectively, typical for post magneto-hydrodynamic ("C")-shock material. These numbers can be compared with the values derived from our methanol modeling in $\$ 5.1$.

\section{OBSERVATIONS}

Our observations were made with the Bell Laboratories $7 \mathrm{~m}$ telescope in the spring of 1995. The beamwidth at $86 \mathrm{GHz}$ is $2^{\prime}$. System temperatures ranged from 300 to $400 \mathrm{~K}$, but could be as high as $1200 \mathrm{~K}$. Generally while mapping, the observing time per point was adjusted to retrieve uniform rms noise values. We observed the lines listed in Table 2 , some of them (as listed in the table caption) with a $256 \times 1 \mathrm{MHz}$ filterbank, others with a $256 \times 250 \mathrm{kHz}$ filterbank.

We mapped only the $\mathrm{SiO}(2-1)$ line extensively over the $l, b \sim 1.0 \times 0.35$-sized region shown in Figs. 1 and 5 . Mostly high-quality spectra of this line and the others listed in Table 2 were taken toward the "fiducial" positions given in Table 3, which are marked in Fig. 1. The $\mathrm{CS}$ and $\mathrm{SiO}$ spectra taken toward positions 1-6 are presented in Fig. 22 and the $\mathrm{CH}_{3} \mathrm{OH}$ spectra in Fig. 3 and 4 . Line parameters, obtained by Gaussian fitting are 
presented in Table 4. Because of the existence of multiple velocity components, large line widths and line blending, Gaussian fitting of the $\mathrm{CH}_{3} \mathrm{OH} 2_{k}-1_{k}$ series was not viable. The interpretation of the methanol results is discussed in $\$ 5.1$.

\section{OUR MOLECULAR LINE DATA}

As shown in Figs. 2-5, we observe emission between velocities of $\sim-10$ and +200

$\mathrm{km} \mathrm{s}^{-1}$. Molecular gas at velocities $<-100 \mathrm{~km} \mathrm{~s}^{-1}$ and $>120 \mathrm{~km} \mathrm{~s}^{-1}$ is usually attributed to the expanding molecular ring (EMR, Kaifu et al. 1972, 1974; Scoville 1972) around the Galactic center. Given its position, G1.6-0.025 is at the high longitude end of that ring. That the emission in all the three molecules observed by us is much more prominent and widespread than in "normal" Galactic disk clouds favors a Galactic center location; see also the arguments brought forward in $\$ 2.1$.

\subsection{SiO Emission Distribution and Velocity Structure}

Figs. 1 and 5 show that the $\mathrm{SiO} J=2-1$ emission is very clumpy on different scales, the smallest of which seem to be resolved by our beam, whose HPBW corresponds to 4.7 pc. This picture is similar to that presented by the $J=1-0$ line mapped by Martin-Pintado et al. (1997) with the same resolution over a similar-sized region extending the area mapped by us to smaller longitudes, i.e., from $l=+0.8$ to $l=-0.2$, covering Sgr B2 and the Galactic center proper (Sgr A). Huettemeister et al. (1998) observed the ${ }^{28} \mathrm{SiO}$ and the ${ }^{29} \mathrm{SiO} J=2-1$ and the ${ }^{28} \mathrm{SiO} 5-4$ transitions toward CS peaks found by Bally et al. (1987). They used LVG calculations to model these two-line, two-isotopomer data to constrain density, temperature and $\mathrm{SiO}$ abundance toward all these positions. Their data are consistent with a hot $T>100 \mathrm{~K}$, low density medium $\sim 10^{4} \mathrm{~cm}^{-3}$, in which, 
particularly, the higher- $J \mathrm{SiO}$ transitions are highly subthermally excited. One of the positions they observed, at $l, b=+1.31,-0.31$, is within $\approx 2^{\prime}$ of our position 6 . Toward this position, they find the highest fractional $\mathrm{SiO}$ abundance $\left(10^{-8}\right)$ of all the 33 positions

they observed, which are spread over the whole of the CMZ. Huettemeister et al. (1998) invoke a shock origin for the elevated $\mathrm{SiO}$ abundance there and also toward other locations for which models of the Galactic bar gravitational potential predict cloud-cloud collisions (see $\$ 4.3$ and 5.2 ).

\subsection{Methanol Emission}

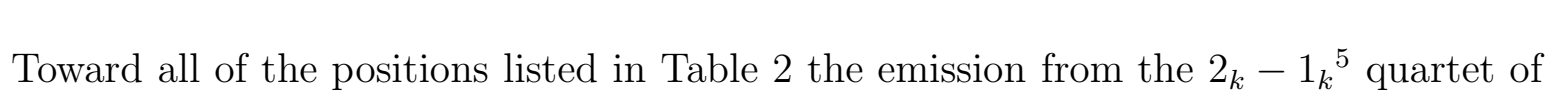
$\mathrm{CH}_{3} \mathrm{OH}$ is stronger than that of the $\mathrm{SiO}$ line, and, amazingly, even stronger than that of the CS line (see Table 4, Figs. 3 and 4). Given their strong blending, it is impossible to determine the properties of these lines by fitting Gaussians in any meaningful way. Instead we performed the model calculations described in \$5.1 to predict intensities for them lines and the $84 \mathrm{GHz} 5_{-1}-4_{0} E$ transition and, in turn, to constrain the physical parameters of the emission region.

\subsection{Evidence for Cloud-Cloud Collisions}

We note that we find a spatial coincidence (at $l, b=1.23,-0.05$ ) of an SiO clump with emission in the $17.6 \mathrm{~km} \mathrm{~s}^{-1}$ channel with one with emission in the $100 \mathrm{~km} \mathrm{~s}^{-1}$ channel.

\footnotetext{
${ }^{5}$ The projection of the angular momentum quantum number, $k$, runs from $-J$ to $+J$ for $E$-type $\mathrm{CH}_{3} \mathrm{OH}$. For $A$-type $\mathrm{CH}_{3} \mathrm{OH}$ a capital $K$ is used, with $0 \leq K \leq J$. When referring in one expression to levels from both species, lowercase $k$ is used.
} 
We also observe a coincidence, at $l, b=1.22,+0.10$, between clumps in the 100.4 and one in $155.6 \mathrm{~km} \mathrm{~s}^{-1}$ channels. Other such coincidences can be found in the channel maps.

A similar coincidence of two clumps with widely different velocities that appear at the same projected area in space has been reported by Haschick \& Baan (1993) who note, in the $4_{-1}-3_{0} E \mathrm{CH}_{3} \mathrm{OH}$ emission, a coincidence of a clump in the $40-70 \mathrm{~km} \mathrm{~s}^{-1}$ velocity interval (which they call Dm) with one in the $150-167 \mathrm{~km} \mathrm{~s}^{-1}$ interval (Em) at $l, b=1.594,+0.015$. This particular spatial coincidence of components at these velocities was also pointed out by Salii et al. (2002), who present a map of the high velocity emission along with the integrated emission in the $5_{-1}-4_{0} E$ and the blended $2_{k}-1_{k}$ lines of $\mathrm{CH}_{3} \mathrm{OH}$ for which we took spectra only toward selected positions. Both the low and the high velocity emission have a similar extent in the $\mathrm{CH}_{3} \mathrm{OH}$ as the $\mathrm{SiO}$ emission (for the high velocity emission, see the $156 \mathrm{~km} \mathrm{~s}^{-1}$ channel map in our Fig,(5).

Haschick \& Baan (1993) credit Sobolev (1992), who interpreted the $4_{-1}-3_{0} E \mathrm{CH}_{3} \mathrm{OH}$ data of Berulis et al. (1992) in a scenario involving a cloud-cloud collision, an intriguing idea, which is further promoted by Salii et al. (2002); see $\$ 5.2$.

\section{PHYSICAL CONDITIONS AND CHEMISTRY IN G1.6-0.025}

\subsection{Methanol Statistical Equilibrium Calculations}

In addition to our own data and those of Salii et al. (2002), further important constraints on $\mathrm{CH}_{3} \mathrm{OH}$ excitation in $\mathrm{G} 1.6-0.025$ come from widespread enhanced absorption (overcooling) in the $12.2 \mathrm{GHz} 2_{0}-3_{-1} E$ line (Whiteoak \& Peng 1989) and the also widespread maser emisson in the $4_{-1}-3_{0} E$ line (Haschick \& Baan 1993; Liechti \& Wilson 1996). As explained, e.g., by Menten (1991) one expects, in the absence of a strong far-infrared field, which is certainly the case in G1.6-0.025, an overpopulation of 
the $k=-1$ ladder relative to the $k=0$. Similarly, one might naively expect overcooling in the $109 \mathrm{GHz} 0_{0}-1_{-1} E$ transition, where we observe neither absorption nor emission with a $3 \sigma$ upper limit of $0.57 \mathrm{~K}$. This absence is explained by our model predictions (see below).

To address these issues quantitatively, we performed model calculations. Leurini et al. (2004) used the rate coefficients for collisions of $\mathrm{CH}_{3} \mathrm{OH}$ with He calculated by Pottage et al. (2002) to investigate the excitation of $\mathrm{CH}_{3} \mathrm{OH}$ over a range of physical parameters typical of star-forming regions. They also presented a new technique to handle the problem of deriving physical parameters of a source from spectroscopic data; the technique is based on the simultaneous fit of multiple lines in a spectrum (when present) with a synthetic spectrum computed using the LVG approach for solving the radiative transfer equations, in the derivation of de Jong et al. (1975). This analysis is particularly well-suited for the case of strongly blended lines, where "by-hand" Gaussian fitting of lines with multiple components often leads to unreliable results. Moreoever, following Cesaroni \& Walmsley (1991), the effect of overlap of lines in the excitation of the $\mathrm{CH}_{3} \mathrm{OH}$ molecule is taken into account defining an average optical depth and brightness temperature for lines with a frequency separation

$$
\nu_{i}-\nu_{j} \leq \Delta \nu_{i}+\Delta \nu_{j}
$$

Using the technique described above, we analyzed our data toward two of the observed positions, namely numbers 2 and 3 of Table [3, to derive the physical parameters of the region. In addition to our own data, we have also compared the predictions of our model for other $\mathrm{CH}_{3} \mathrm{OH}$ lines observed toward position 2 by other authors. Our model calculations do not predict absorption in the $0_{0}-1_{-1} E$ transition for a wide range of physical parameters ( $n$ from $10^{3}$ to $10^{8} \mathrm{~cm}^{-3} ; T_{k i n}$ from 10 to $200 \mathrm{~K} ; N\left(\mathrm{CH}_{3} \mathrm{OH}\right.$ ) from $10^{12}$ to $10^{16} \mathrm{~cm}^{-2}$ ). The simultaneous modeling of the $2_{k}-1_{k}$ and $5_{-1}-4_{0} E$ lines gives constraints on the column density of methanol and on the $\mathrm{H}_{2}$ density. The $5_{-1}-4_{0} E$ transition is strongly inverted over a wide range of physical parameters, for $n\left(\mathrm{H}_{2}\right)>10^{4} \mathrm{~cm}^{-3}$ and $\mathrm{CH}_{3} \mathrm{OH}$ column 
densities higher than $10^{15} \mathrm{~cm}^{-2}$ (see Fig. 8). Hence, the non-detection of obvious strong maser action (but see below) in our observations indicates low values for the column density of the gas. The $2_{k}-1_{k}$ lines are, on the other hand, sensitive to the density of the gas (see Fig. 4 of Leurini et al. (2004)).

Inspecting the $2_{k}-1_{k} \mathrm{CH}_{3} \mathrm{OH}$ map presented by Salii et al. (2002) we assumed the $\mathrm{CH}_{3} \mathrm{OH}$ emission to be extended compared to the $7 \mathrm{~m}$ telescope beam. We modeled the data with two velocity components, corresponding to the high velocity clump and to the extended cloud. The LVG fit overlaid on the data is shown in Figs. 6 and 7 . The results determined from the fit are given in Table 5. Table 6] shows our model predictions for the other $\mathrm{CH}_{3} \mathrm{OH}$ transitions observed by other authors. Pointing and absolute calibration uncertainties and beam-size differences can make the determination of physical parameters less reliable when comparing data from different telescopes; in our case however, beam-size differences should not affect the results as, given the source sizes, beam filling factors for the different transitions are pretty close to 1.

Salii et al. (2002) determined the physical parameters toward our position 2 by analyzing several methanol transitions. Their results do differ somewhat from ours. In particular, they find somewhat smaller spatial densities and $\mathrm{CH}_{3} \mathrm{OH}$ column densities. From their analysis they conclude that the high velocity clump has a hydrogen density, $n\left(\mathrm{H}_{2}\right)$, less than $10^{4} \mathrm{~cm}^{-3}$, column densities between $4 \times 10^{11}$ and $6 \times 10^{12} \mathrm{~cm}^{-2}$ and temperatures in the 150-200 K range. For the extended cloud they infer a hydrogen density $<10^{6} \mathrm{~cm}^{-3}$, column densities larger than $6 \times 10^{11} \mathrm{~cm}^{-2}$ and a kinetic temperature of less than $80 \mathrm{~K}$. Assuming that all the gas is in molecular form, this translates in spatial densities of molecular hydrogen less than $3 \times 10^{3} \mathrm{~cm}^{-3}$ for the extended cloud and $n\left(\mathrm{H}_{2}\right)<5 \times 10^{3} \mathrm{~cm}^{-3}$ for the high velocity clump. With these parameters, they can reproduce the observed line intensities for several transitions, but fail to explain the simultaneous deep absorptions in 
the $2_{0}-3_{-1} E$ and $2_{1}-3_{0} A^{+}$lines at $12.18 \mathrm{GHz}$ and $156.6 \mathrm{GHz}$, and the brightness of the emission in the $J_{0}-J_{-1} E, J=1,2,3$ blend. They conclude that the absorptions and the $J_{0}-J_{-1} E, J=1,2,3$ blended emission come from different parts of the cloud.

Our model predictions (see Table 6) overestimate the $J_{0}-J_{-1} E, J=1,2,3$ blended emission and underestimate the absorption in the $2_{0}-3_{-1^{-}} E$ transition in the extended cloud, but can reproduce the simultaneous absorptions and the emission in the $J_{0}-J_{-1} E$ blend. For the high velocity clump, our predictions are in good agreement with the observations. Moreover, spatial densities of the order of a few $10^{3} \mathrm{~cm}^{-3}$, as inferred by Salii et al. (2002), fail to reproduce our observations of the $5_{-1}-4_{0}-E$ line.

At position 2, Haschick \& Baan (1993) find maser emission in the $4_{-1}-3_{0}-E$ line on top of a broad thermal-looking component. Fitting the maser component in our model would need a second, narrower component for both the high velocity clump and the extended cloud, which is not detected in our observations. Therefore we do not include in our analysis any other component to account for the narrow maser features in the $4_{-1}-3_{0} E$ line. However, the maser action in the $4_{-1}-3_{0} E$ and the simultaneous absence of it in the $5_{-1}-4_{0} E$ line can give interesting constraints for the physical parameters of the regions. Leurini et al. (in prep.) have extensively analyzed the pumping mechanisms of class I $\mathrm{CH}_{3} \mathrm{OH}$ masers. Their calculations confirm collisions to be responsible for the excitation of class I masers and suggest the maser action in these lines to be used as a density indicator. Both the $4_{-1}-3_{0}-E$ and $5_{-1}-4_{0} E$ transitions are inverted at low densities; however, the inversion of $5_{-1}-4_{0} E$ line starts with $n\left(\mathrm{H}_{2}\right)>10^{4} \mathrm{~cm}^{-3}$ with $\mathrm{CH}_{3} \mathrm{OH}$ column densities higher than $10^{15} \mathrm{~cm}^{-2}$, while the $4_{-1}-3_{0} E$ line mases also at lower densities, as Fig. 9 shows, almost independently from the kinetic temperature. The above is also found by Berulis et al. (1992) and Sobolev (1992). At lower column densities, the inversion of the $5_{-1}-4_{0} E$ line happens at slightly higher densities. Therefore the detection of a maser 
component at $36 \mathrm{GHz}$ together with the non-detection of strong maser action manifested by narrow features in the $84.5 \mathrm{GHz}$ line puts an upper limit on of $\sim 10^{4}-10^{5} \mathrm{~cm}^{-3}$ on the spatial hydrogen density of the region, depending on the column density of methanol.

Nevertheless, comparison of $5_{-1}-4_{0} E$ and $2_{k}-1_{k}$ spectra (Figs. 3 and 4 ) shows that the former generally are narrower than the latter, which might at first look be interpreted as line narrowing accompanying maser action. However, since some of the (noisy) spectra also cover incongruent velocity ranges this might actually not be the cause for this difference in appearance. Another possibility would be the existence of two gas components in the beam with different densities and/or temperatures, which might contribute to the lines in question in different proportions. This could explain the narrower line profiles of the $5_{-1}-4_{0} E$ line, which seems not to be excited in one of the velocity components, and the underestimate of line intensity in the model. However, the maps of the $5_{-1}-4_{0} E$ and $2_{k}-1_{k}$ lines published by Salii et al. (2002) (and with a spatial resolution of $\sim 57^{\prime \prime}$ ), do not show any large discrepancies between the distributions of the two lines. Needless to say, the described discrepancy illustrates the qualitative nature of our results.

To summarize, our statistical equilibrium modeling of the $\mathrm{CH}_{3} \mathrm{OH}$ lines observed by us and others indicates relatively high densities $\left(>6 \times 10^{4} \mathrm{~cm}^{-3}\right)$ and moderate temperatures $(30-60 \mathrm{~K})$ for two representative positions in the G1.6-0.025 cloud at $\mathrm{v}_{\mathrm{LSR}}=50 \mathrm{~km} \mathrm{~s}^{-1}$. In the high velocity component, lower densities are inferred by the model for both positions; for position 2, a high temperature $(190 \mathrm{~K})$ is needed to reproduce the observations, while cold gas $(16 \mathrm{~K})$ is needed for position 3. The latter is puzzling as, in analogy to position 2, one might also expect an enhanced temperature for high velocity $\mathrm{CH}_{3} \mathrm{OH}$ emission. High velocity $\mathrm{SiO}$ is not even detected toward position 3 and high velocity CS barely. Methanol abundances relative to $\mathrm{H}_{2}$ are of the order of $10^{-7}-10^{-8}$. Finally, we note that the temperatures and densities we derive are of the same order of the values that 
Whiteoak \& Peng (1989) derive from their modeling of the $\mathrm{CH}_{3} \mathrm{OH} 2_{0}-3_{-1} E$ line.

Huettemeister et al. (1993) conducted a multi-transition study of $\mathrm{NH}_{3}$ inversion lines with energies of up to $408 \mathrm{~K}$ above ground toward 36 positions in the CMZ. They find evidence for two components pervasive throughout the CMZ, both at each position at roughly the same velocity, a cool one, $T \sim 25 \mathrm{~K}$ and a hot one $\sim 200 \mathrm{~K}$. The densities of the these components are not well constrained by their observations, but they argue that the hot and cool gas have densities of $10^{4}$ and $10^{5} \mathrm{~cm}^{-3}$, respectively.

In their study, they also included one position within the area that we mapped in $\mathrm{SiO}$ emission $(l, b=+1.15,-0.09)$ and several others just abutting it; i.e., within 0.2 degrees outside of its boundaries. Since they do not present data toward the positions toward which we see a high(=clump)- and a low(= general cloud $)$-velocity component, a direct comparison with our analysis is difficult. However, we can say that we do not see evidence for a high temperature component at the general cloud velocities toward the two positions that we analyzed, while they find evidence for both a cool and hot component at these velocities.

The reason why we do not see a hot component may be a selection effect based on our choice of lines. The rotation temperatures Huettemeister et al. (1993) derive from their lowest excitation lines alone $[J, K=(1,1)$ and $(2,2)$ at energies of 23 and $64 \mathrm{~K}$ above ground, respectively] invariably are between 18 and $40 \mathrm{~K}$ throughout the CMZ and mostly around $25 \mathrm{~K}$. In contrast, rotation temperatures determined from the $(4,4)$ and $(5,5)$ lines (at 200 and $295 \mathrm{~K}$ above ground, respectively) are all $>50 \mathrm{~K}$, most $>80 \mathrm{~K}$, and some as high as 200 K. Since we didn't observe any high excitation lines, given the experience with $\mathrm{NH}_{3}$, we could not detect any hot component in G1.6-0.025 at velocities at which emission from cold as well as from hot gas is arising, but only at velocities at which only the hot component emits (i.e., high velocity clumps). 
To estimate the column density of molecular hydrogen and derive the $\left[\mathrm{CH}_{3} \mathrm{OH}\right] /\left[\mathrm{H}_{2}\right]$ ratio, we used the ${ }^{13} \mathrm{CO} J=1-0$ data imaged by Bally et al. (1987) with the Bell Labs $7 \mathrm{~m}$ telescope. Assuming the Local Thermal Equilibrium approximation, and using the kinetic temperatures derived from the analysis of the methanol emission, we computed the ${ }^{13} \mathrm{CO}$ column densities for positions 1 and 2, for the high velocity clump and for the extended cloud, by integrating over the velocity channels corresponding to the $\mathrm{CH}_{3} \mathrm{OH}$ emission. To convert the ${ }^{13} \mathrm{CO}$ column density into a $\mathrm{H}_{2}$ column density, we used a typical abundance relative to $\mathrm{H}_{2}$ of $10^{-4}$ for $\mathrm{CO}$ and a value of 20 for the ${ }^{12} \mathrm{C} /{ }^{13} \mathrm{C}$ isotopic ratio. This is the value Wilson \& Rood (1994) give for the ${ }^{12} \mathrm{C} /{ }^{13} \mathrm{C}$ ratio for molecular clouds in the Galactic center region. If we, however, used 11 for the latter ratio, which Gardner \& Whiteoak (1981) derive for G1.6-0.025 from observations of formaldehyde isotopomers, the relative methanol abundance ratios given in the following would increase by a factor of 2 .

Assuming that the column density of $\mathrm{CH}_{3} \mathrm{OH}-\mathrm{A}$ and $\mathrm{CH}_{3} \mathrm{OH}-\mathrm{E}$ are the same, our derived column densities translate into abundances relative to molecular hydrogen of $10^{-8}$ for the high velocity clump and $1 \times 10^{-6}$ for the extended cloud toward position 2 and to $10^{-8}$ and $3 \times 10^{-8}$, respectively, for position 3 . The high velocity component is not detected in the ${ }^{13} \mathrm{CO}$ line toward position 3 and we could only derive an upper limit to the $\mathrm{H}_{2}$ column density based on the rms noise value of the data. Therefore, the $\mathrm{CH}_{3} \mathrm{OH}$ abundance ratio estimated for this component is a lower limit to its true value.

\subsection{Shock Chemistry in G1.6-0.025}

As discussed in $\S \$ 4.1$ and 5.1 the $\mathrm{SiO}$ and $\mathrm{CH}_{3} \mathrm{OH}$ abundances are enhanced relative to cold molecular cloud values. The observed chemical peculiarities of G1.6-0.025 could be the the result of several mechanisms all involving shocks: cloud-cloud collisions (see \$4.1), a molecular cloud-SNR interaction, and whatever is responsible for the peculiar large 
linewidths in GC GMCs in general (increased turbulence, tidal forces).

It is tempting to assume that the high observed $\mathrm{SiO}$ and $\mathrm{CH}_{3} \mathrm{OH}$ abundances in G1.6-0.025 have a common origin. With regard to these two species, a picture similar to G1.6-0.025 presents itself in the molecular peak M-0.02 - 0.07 (the so-called "20 $\mathrm{km} \mathrm{s}^{-1}$ cloud"), which lies $\sim 2^{\prime}$ NEE of the Galactic center radio source Sgr $A^{\star}$ at a position where the supernova remnant Sgr A East (SNR G0.0+0.0) appears to interact with a molecular cloud. Here, Martin-Pintado et al. (1997) find strong $\operatorname{SiO}(J=1-0)$ emission, while Szczepanski et al. (1989) and, at higher resolution, Liechti \& Wilson (1996) find very strong maser emission in the $36 \mathrm{GHz} 4_{-1}-3_{0} E$ line of methanol. Just like in G1.6-0.025 the $\mathrm{CH}_{3} \mathrm{OH}$ emission consists of a few narrow spikes and intense "broad" emission. $1720 \mathrm{MHz} \mathrm{OH}$ masers are also found in this region on the near-side of the $\mathrm{SiO} / \mathrm{CH}_{3} \mathrm{OH}$ emission distribution relative to Sgr A East facing the SNR (Karlsson et al. 2003; Pihlström \& Siouwerman 2006; Yusef-Zadeh et al. 2007). We emphasize the similarity to G1.6-0.025, with the SNR $1.4-0.1$ (see \$2.3), projected on and possibly interacting with it.

The existence of $1720 \mathrm{MHz} \mathrm{OH}$ maser emission argues, as discussed in \$2.3, also for (C-)shock. The densities and temperature we derive from the methanol lines are very similar to the values necessary for $1720 \mathrm{MHz} \mathrm{OH}$ maser emission as discussed there. The chemistry in the molecular gas interacting with the SNR IC 443 was studied by van Dishoeck et al. (1993). One subregion, clump G I, was found to show a particularly rich chemical picture. However, one dramatic difference between the molecular content of G1.6-0.025 and molecular IC 443 clump G I a is the complete dearth of any $\mathrm{CH}_{3} \mathrm{OH}$ in the latter. van Dishoeck et al. (1993) give a relatively sensitive $2 \sigma$ upper limit of $0.2 \mathrm{~K}$ for any line in the $241.7 \mathrm{GHz} \mathrm{CH}_{3} \mathrm{OH} 5_{k}-4_{k}$ series, while various $\mathrm{SiO}$ lines are two to three times stronger than that. 
As for $\mathrm{SiO}$, high abundances after the passage of magnetohydrodynamic C-shocks can result from a combination of the setting free of $\mathrm{SiO}$ into the gas-phase by sputtering of the (charged, and hence coupled to the magnetic field) grain cores by neutral particles in the region of the C-shock where the relative velocities between charged and neutral particles are large, and following gas-phase reactions (Schilke et al. 1997). This is true for pristine material, where silicon resides in the grain cores. If, as is the case in the Galactic center, molecular clouds are frequently exposed to cloud-cloud collisions, silicon may, after the initial release from the core, reside either in the gas phase (possibly some of it in $\mathrm{SiO}_{2}$ as suggested by Schilke et al. (1997)) or in grain mantles, with a lower binding energy.

How is the methanol produced in shocks? Gas-phase production of methanol has been shown to be insufficient to create the observed abundances in dark, quiescent clouds by many orders of magnitude (Geppert et al. 2005). Grain surface production however seems to be efficient, as shown by Hidaka et al. (2004). This indeed is the proposed mechanism for producing the high methanol abundances found in "hot cores" around high-mass young (proto)stars, where $\mathrm{CH}_{3} \mathrm{OH}$ is created by the evaporation from grain mantles once temperatures exceed $\sim 100 \mathrm{~K}$. Shocks also would be able to release material from grain mantles, either by sputtering, even at lower shock speeds than needed for $\mathrm{SiO}$ production, since here the more weakly bound ice mantles have to be destroyed instead of the grain cores, as needed for $\mathrm{SiO}$; or by thermal evaporation in the hot shocked gas. This mechanism has indeed been evoked for explaining the high methanol abundances in shocked protostellar outflows (e.g., Bachiller \& Perez Gutierrez 1997).

To evaporate methanol from ice mantles, however, these ice mantles have to be present. While there is observational evidence from IR spectroscopy that ice mantles do exist in the cold envelopes of protostars (to be released by outflows or heating by the igniting star), the conditions for formation of ice mantles in the highly turbulent, warm and relatively low 
density environment of Galactic center clouds (of which G1.6-0.025 is a member) seem less favorable. Deciding if sufficient methanol abundances in ice mantles can be built up under these conditions will require detailed modeling.

Another option could be that under GC cloud conditions, ice mantles do not build up, but $\mathrm{CO}$ can reside long enough on the grain surface to be transformed into $\mathrm{CH}_{3} \mathrm{OH}$, which then is desorbed either by sputtering in vortices, or just thermally desorbed. In this case, the elevated methanol abundance would not be related to any specific shock event, but be the steady state abundance under these special conditions. Modeling would certainly be illuminating, but an observational consequence of this mechanism would be a uniformly high methanol abundance in the Galactic center, which may actually apply (Requena-Torres et al. 2006).

Alternatively, in the elevated temperatures of a C-shock, $\mathrm{CH}_{3} \mathrm{OH}$ may be created by the endothermic gas-phase reactions

$$
\mathrm{CH}_{4}+\mathrm{OH} \rightarrow \mathrm{CH}_{3} \mathrm{OH}+\mathrm{H}
$$

and

$$
\mathrm{CH}_{3}+\mathrm{H}_{2} \mathrm{O} \rightarrow \mathrm{CH}_{3} \mathrm{OH}+\mathrm{H}
$$

discussed by Hartquist et al. (1995), which have endothermicities of 6500 and $14700 \mathrm{~K}$, respectively. The abundances of reaction partners $\mathrm{OH}$ and $\mathrm{H}_{2} \mathrm{O}$ certainly would be enhanced in a shock, but to judge these reactions' importance would require running shock models, looking also carefully at possible shock destruction mechanisms for $\mathrm{CH}_{3} \mathrm{OH}$. Observationally, this would, just as the option of removing $\mathrm{CH}_{3} \mathrm{OH}$ from ice mantles, imply a correlation of elevated $\mathrm{CH}_{3} \mathrm{OH}$ abundances with shock events.

In practice, it will be very hard to distinguish observationally between these scenarios, because $\mathrm{SiO}$, the canonical shock tracer, is so widely distributed. This suggests that either 
shocks are ubiquitous, or that the grains are processed to a degree that a significant fraction of silicon resides in more weakly bound form either in the gas phase or on the grain surface, so that the release mechanisms for $\mathrm{SiO}$ and $\mathrm{CH}_{3} \mathrm{OH}$ are similar.

While we argue above that the interaction with a SNR may influence the chemistry in at least part of G1.6-0.025, cloud-cloud collisions resulting from the special dynamics induced by the Galactic bar potential may play a major role for G1.6-0.025 and other GC clouds as a whole (see Huettemeister et al. 1998; Rodriguez-Fernandez et al. 2006).

\section{G1.6-0.025 IS AT THE OUTER REACHES OF THE GALACTIC CENTER ORGANIC MOLECULE REGION}

There actually is evidence for a giant repository of organic molecules coextensive with the Central Molecular Zone, of which G1.6-0.025 demarcates the high-longitude border (Menten 2004).

The first evidence for extended organic material in the CMZ came from widespread 4.8 GHz H${ }_{2} \mathrm{CO}$ absorption (Scoville et al. 1972); see also Zvlka et al. (1992). Given the ubiquity of formaldehyde in molecular clouds (e.g. Downes et al. 1980), one might dismiss this "as nothing special". $\mathrm{CH}_{3} \mathrm{OH}$, on the other hand, has usually quite low abundance and is difficult to detect outside hot, dense cloud cores. Nevertheless, Gottlieb et al. (1979) find the $834 \mathrm{MHz}\left(1_{1}-1_{1}\right) A^{\mp}$ line in the CMZ in emission and extended relative to their $40^{\prime}($ !) beam, concluding it is (weakly) inverted and amplifying the strong background radio emission.

Other molecules similarly (or even) more complex than $\mathrm{CH}_{3} \mathrm{OH}$ and $\mathrm{H}_{2} \mathrm{CO}$ were found widespread throughout the $\mathrm{CMZ}$, such as $\mathrm{HCOOH}$ and $\mathrm{C}_{2} \mathrm{H}_{5} \mathrm{OH}$ (Minh et al. 1992; Martín-Pintado et al. 2001) and, very recently, $\left(\mathrm{CH}_{3}\right)_{2} \mathrm{O}, \mathrm{HCOOCH}_{3}, \mathrm{HCOOH}$, and 
$\mathrm{CH}_{3} \mathrm{COOH}($ Requena-Torres et al. 2006$)$

Furthermore, mapping of the $\mathrm{HNCO} 5_{05}-4_{04}$ transition (made serendipitously simultaneously with a $\mathrm{C}^{18} \mathrm{O}$ survey), shows that the emission in this line is extending continuously from $l=-0.2$ to +1.7 , right out to G1.6-0.025! (Dahmen et al. 1997). The possible existence of such a huge mass of organic material in our Galactic center is extremely exciting and its extent, chemistry, and excitation should be studied with suitable tracers.

In fact, G1.6-0.025 coincides with the third-strongest peak in the integrated HNCO distribution (after the general Sgr B2 region and an area around $l=1 \mathrm{deg}, b=0 .^{\circ}$ ) and the ratio of the integrated intensities of the $\mathrm{HNCO}$ to that of the $\mathrm{C}^{18} \mathrm{O}$ line in $\mathrm{G} 1.6-0.025$ is the highest in the whole CMZ. Maybe it the low UV radiation field density resulting from the absence of young high mass stars in G1.6-0.025 is conducive to the existence of (fragile) complex molecules. Given this, one might also expect other complex molecules than $\mathrm{CH}_{3} \mathrm{OH}$ and $\mathrm{HNCO}$ to have large abundances in G1.6-0.025. Possibly the best spectral range to search for those is the $3 \mathrm{~mm}$ window since, given our density estimates (\$5.1), submillimeter lines might have prohibitively high critical densities.

\section{CONCLUSIONS}

Here we summarize our main conclusions. We find very little evidence for star-formation in over the whole $\sim 0.2 \mathrm{deg}^{2}$ region of the G1.6-0.025 GMC. Our large scale, medium resolution $\left(2^{\prime}\right)$ mapping in the $J=2-1$ transition of SiO reveals clumpy emission over an $\sim 0.6 \times 0.3$ region stretching along the Galactic plane. Toward selected positions, we have observed emission in the $2_{k}-1_{k}$ quartet of $\mathrm{CH}_{3} \mathrm{OH}$ lines and the $\mathrm{CS} 2-1$ line. Toward all of these, the $\mathrm{CH}_{3} \mathrm{OH}$ lines are several times stronger than both the $\mathrm{CS}$ and the $\mathrm{SiO}$ 
lines. In addition, spectra of other methanol lines where taken. The wide widths of all the observed lines, similar to values generally found in the Galactic center, indicates a high degree of turbulence. A high velocity clump with $\mathrm{a} \sim 100 \mathrm{~km} \mathrm{~s}^{-1}$ higher velocity than the molecular cloud may indicate a cloud-cloud collision. Statistical equilibrium modeling of all the $\mathrm{CH}_{3} \mathrm{OH}$ lines observed by us and others indicates relatively high densities and moderate temperatures for one representative position in the G1.6-0.025 cloud $\left(810^{4} \mathrm{~cm}^{-3} / 30 \mathrm{~K}\right)$ and higher temperature $(190 \mathrm{~K})$, but lower density $\left(410^{4} \mathrm{~cm}^{-3}\right.$ in the high velocity clump. For a second position we also find densities of several times $10^{4} \mathrm{~cm}^{-3}$ for both the low and the high velocity emission, but a puzzling low temperature for the high velocity clump $(16 \mathrm{~K})$ and warmer gas $(60 \mathrm{~K})$ for the low velocity $50 \mathrm{~km} \mathrm{~s}^{-1}$ gas. Different scenarios are possible in which shock chemistry might enhance the $\mathrm{CH}_{3} \mathrm{OH}$ and $\mathrm{SiO}$ abundances in G1.6-0.025 and elsewhere in the Central Molecular Zone by grain-gas chemistry or by hot gas chemistry.

We would like to thank Darek Lis for fitting the FIR/submm spectral energy distributions, Maria Messineo and Harm Habing for discussions of OH/IR stars, and Malcolm Walmsley for comments on the manuscript. 


\section{REFERENCES}

Bachiller, R. \& Perez Gutierrez, M. 1997, Ap. J. (Letters) , 487, L93+

Bally, J., Stark, A. A., Wilson, R. W., \& Henkel, C. 1987, ApJS, 65, 13

-. 1988, ApJ, 324, 223

Batrla, W., Matthews, H. E., Menten, K. M., \& Walmsley, C. M. 1987, Nature, 326, 49

Becker, R. H., White, R. L., Helfand, D. J., \& Zoonematkermani, S. 1994, ApJS, 91, 347

Berulis, I. I., Kalenski, S. V., Sobolev, A. M., \& Strelnitski, V. S. 1992, Astronomical and Astrophysical Transactions, 1, 231

Caswell, J. L. 1996, MNRAS, 283, 606

Cesaroni, R. \& Walmsley, C. M. 1991, A\&A, 241, 537

Dahmen, G., Huttemeister, S., Wilson, T. L., \& Mauersberger, R. 1998, A\&A, 331, 959

Dahmen, G., Huttemeister, S., Wilson, T. L., Mauersberger, R., Linhart, A., Bronfman, L., Tieftrunk, A. R., Meyer, K., Widenhover, W., Dame, T. M., Palmer, E. S., May, J., Aparici, J., \& Macauliffe, F. 1997, A\&AS, 125, 1

de Jong, T., Dalgarno, A., \& Chu, S.-I. 1975, ApJ, 199, 69

Downes, D., Wilson, T. L., Bieging, J., \& Wink, J. 1980, A\&AS, 40, 379

Figer, D. F., McLean, I. S., \& Morris, M. 1999, ApJ, 514, 202

Figer, D. F., Najarro, F., Gilmore, D., Morris, M., Kim, S. S., Serabyn, E., McLean, I. S., Gilbert, A. M., Graham, J. R., Larkin, J. E., Levenson, N. A., \& Teplitz, H. I. 2002, ApJ, 581, 258 
Figer, D. F., Rich, R. M., Kim, S. S., Morris, M., \& Serabyn, E. 2004, ApJ, 601, 319

Gardner, F. F. \& Boes, F. 1987, Proceedings of the Astronomical Society of Australia, 7, 185

Gardner, F. F. \& Whiteoak, J. B. 1981, MNRAS, 194, 37P

-. 1982, MNRAS, 199, 23P

Gardner, F. F., Whiteoak, J. B., Forster, J. R., Peters, W. L., \& Kuiper, T. B. H. 1985, Proceedings of the Astronomical Society of Australia, 6, 176

Garrod, R. T. \& Herbst, E. 2006, A\&A, 457, 927

Geppert, W. D., Hellberg, F., Österdahl, F., Semaniak, J., Millar, T. J., Roberts, H., Thomas, R. D., Hamberg, M., Ugglas, M. A., Ehlerding, A., Zhaunerchyk, V., Kaminska, M., \& Larsson, M. 2005, in IAU Symposium, Vol. 231, Astrochemistry: Recent Successes and Current Challenges, ed. D. C. Lis, G. A. Blake, \& E. Herbst, $117-124$

Gottlieb, C. A., Ball, J. A., Gottlieb, E. W., \& Dickinson, D. F. 1979, ApJ, 227, 422

Güsten, R. \& Downes, D. 1981, A\&A, 99, 27

Güsten, R. \& Philipp, S. D. 2004, in The Dense Interstellar Medium in Galaxies, ed. S. Pfalzner, C. Kramer, C. Staubmeier, \& A. Heithausen, 253-+

Güsten, R., Walmsley, C. M., \& Pauls, T. 1981, A\&A, 103, 197

Hartquist, T. W., Menten, K. M., Lepp, S., \& Dalgarno, A. 1995, MNRAS, 272, 184

Haschick, A. D. \& Baan, W. A. 1993, ApJ, 410, 663

Hidaka, H., Watanabe, N., Shiraki, T., Nagaoka, A., \& Kouchi, A. 2004, ApJ, 614, 1124 
Huettemeister, S., Dahmen, G., Mauersberger, R., Henkel, C., Wilson, T. L., \& Martin-Pintado, J. 1998, A\&A, 334, 646

Huettemeister, S., Wilson, T. L., Bania, T. M., \& Martin-Pintado, J. 1993, A\&A, 280, 255

Kaifu, N., Iguchi, T., \& Kato, T. 1974, Pub. Astr. Soc. Japan , 26, 117

Kaifu, N., Kato, T., \& Iguchi, T. 1972, Nature, 238, 105

Karlsson, R., Sjouwerman, L. O., Sandqvist, A., \& Whiteoak, J. B. 2003, A\&A, 403, 1011

Kuiper, T. B. H., Whiteoak, J. B., Peng, R.-S., Peters, III, W. L., \& Reynolds, J. E. 1993, Ap. J. (Letters) , 416, L33+

Leurini, S., Schilke, P., Menten, K. M., Flower, D. R., Pottage, J. T., \& Xu, L.-H. 2004, A\&A, 422, 573

Liechti, S. \& Wilson, T. L. 1996, A\&A, 314, 615

Lis, D. C. \& Carlstrom, J. E. 1994, ApJ, 424, 189

Lis, D. C. \& Menten, K. M. 1998, ApJ, 507, 794

Lis, D. C., Menten, K. M., Serabyn, E., \& Zylka, R. 1994, Ap. J. (Letters), 423, L39

Liszt, H. S. 1992, ApJS, 82, 495

Lockett, P., Gauthier, E., \& Elitzur, M. 1999, ApJ, 511, 235

Martin, C. L., Walsh, W. M., Xiao, K., Lane, A. P., Walker, C. K., \& Stark, A. A. 2004, ApJS, 150, 239

Martin-Pintado, J., de Vicente, P., Fuente, A., \& Planesas, P. 1997, Ap. J. (Letters) , 482, L45+ 
Martín-Pintado, J., Rizzo, J. R., de Vicente, P., Rodríguez-Fernández, N. J., \& Fuente, A. 2001, Ap. J. (Letters) , 548, L65

Menten, K. 1991, in Astronomical Society of the Pacific Conference Series, Vol. 16, Atoms, Ions and Molecules: New Results in Spectral Line Astrophysics, ed. A. D. Haschick \& P. T. P. Ho, 119-+

Menten, K. M. 2004, in The Dense Interstellar Medium in Galaxies, ed. S. Pfalzner, C. Kramer, C. Staubmeier, \& A. Heithausen, 69-+

Menten, K. M. \& Reid, M. J. 1995, Ap. J. (Letters) , 445, L157

Mezger, P. G., Smith, L. F., \& Churchwell, E. 1974, A\&A, 32, 269

Minh, Y. C., Irvine, W. M., \& Friberg, P. 1992, A\&A, 258, 489

Morris, M. \& Serabyn, E. 1996, ARA\&A, 34, 645

Motte, F., Schilke, P., \& Lis, D. C. 2003, ApJ, 582, 277

Oka, T., Hasegawa, T., Sato, F., Tsuboi, M., \& Miyazaki, A. 1998, ApJS, 118, 455

Panagia, N. 1973, AJ, 78, 929

Pierce-Price, D., Richer, J. S., Greaves, J. S., Holland, W. S., Jenness, T., Lasenby, A. N., White, G. J., Matthews, H. E., Ward-Thompson, D., Dent, W. R. F., Zylka, R., Mezger, P., Hasegawa, T., Oka, T., Omont, A., \& Gilmore, G. 2000, Ap. J. (Letters) , 545, L121

Pihlström, Y. M. \& Sjouwerman, L. O. 2006, Journal of Physics Conference Series, 54, 77

Pottage, J. T., Flower, D. R., \& Davis, S. L. 2002, Journal of Physics B Atomic Molecular Physics, 35, 2541 
Reid, M. J. 1993, ARA\&A, 31, 345

Requena-Torres, M. A., Martín-Pintado, J., Rodríguez-Franco, A., Martín, S., RodríguezFernández, N. J., \& de Vicente, P. 2006, A\&A, 455, 971

Rodriguez-Fernandez, N. J., Combes, F., Martin-Pintado, J., Wilson, T. L., \& Apponi, A. 2006, A\&A, 455, 963

Salii, S. V., Sobolev, A. M., \& Kalinina, N. D. 2002, Astronomy Reports, 46, 955

Schilke, P., Walmsley, C. M., Pineau des Forets, G., \& Flower, D. R. 1997, A\&A, 321, 293

Schuller, F., Omont, A., Felli, M., Testi, L., Bertoldi, F., \& Menten, K. M. 2005, in ESA Special Publication, Vol. 577, ESA Special Publication, ed. A. Wilson, 235-238

Scoville, N. Z. 1972, Ap. J. (Letters), 175, L127+

Scoville, N. Z., Solomon, P. M., \& Thaddeus, P. 1972, ApJ, 172, 335

Sobolev, A. M. 1992, Soviet Astronomy, 36, 590

Szczepanski, J. C., Ho, P. T. P., Haschick, A. D., \& Baan, W. A. 1989, in IAU Symposium, Vol. 136, The Center of the Galaxy, ed. M. Morris, 383-+

van der Veen, W. E. C. J. \& Habing, H. J. 1988, A\&A, 194, 125

van Dishoeck, E. F., Jansen, D. J., \& Phillips, T. G. 1993, A\&A, 279, 541

Walmsley, C. M., Guesten, R., Angerhofer, P., Churchwell, E., \& Mundy, L. 1986, A\&A, 155,129

Walmsley, C. M., Menten, K. M., Batrla, W., \& Matthews, H. E. 1988, A\&A, 197, 271

Whiteoak, J. B. \& Peng, R.-S. 1989, MNRAS, 239, 677 
Wilson, T. L. \& Rood, R. 1994, ARA\&A, 32, 191

Yusef-Zadeh, F., Arendt, R. G., Heinke, C. O., Hinz, J. L., Hewitt, J. W., Pratap, P., Ramirez, S. V., Rieke, G. H., Roberts, D. A., Stolovy, S. R., Wardle, M., \& Whitney, B. A. 2007 , ArXiv e-prints, 705

Yusef-Zadeh, F., Roberts, D. A., Goss, W. M., Frail, D. A., \& Green, A. J. 1999, ApJ, 512, 230

Zylka, R., Guesten, R., Henkel, C., \& Batrla, W. 1992, A\&AS, 96, 525 
Table 1. Radio- and Far-Infrared-Wavelength Properties of Sgr D 7 and D 8

\begin{tabular}{|c|c|c|c|}
\hline Radio name & Sgr D 7 & Sgr D 8 & References \\
\hline$\alpha_{B 19500}$ & 174501.3 & 174547.1 & 1,2 \\
\hline$\delta_{B 1950}$ & -274217 & -274345 & 1,2 \\
\hline$l$ & 1.330 & 1.397 & 1,2 \\
\hline$b$ & 0.051 & -0.006 & 1,2 \\
\hline$S(1.4 \mathrm{GHz})(\mathrm{mJy})$ & - & 26 & 1 \\
\hline$S(1.6 \mathrm{GHz})(\mathrm{mJy})$ & 222 & 21 & 2 \\
\hline$S(5 \mathrm{GHz})(\mathrm{mJy})$ & - & 24.2 & 1 \\
\hline IRAS Name & $17450-2742$ & $17457-2743$ & 3 \\
\hline$S(12 \mu \mathrm{m})(\mathrm{Jy})$ & 9.79: & $5.54 \mathrm{~L}$ & 3 \\
\hline$S(25 \mu \mathrm{m})(\mathrm{Jy})$ & 28.30 & 3.13 & 3 \\
\hline$S(60 \mu \mathrm{m})(\mathrm{Jy})$ & 462.40: & 85.82: & 3 \\
\hline$S(100 \mu \mathrm{m})(\mathrm{Jy})$ & 782.90: & $386.60 \mathrm{~L}$ & 3 \\
\hline$T_{\mathrm{D}, 25-60}(\mathrm{~K})$ & 47 & 43.5 & 4 \\
\hline$T_{\mathrm{D}, 25-100}(\mathrm{~K})$ & 43.5 & 38.5 & 4 \\
\hline$L_{25-60}\left(L_{\odot}\right)$ & $2.6 \times 10^{4}$ & $4.5 \times 10^{3}$ & 4 \\
\hline$L_{25-100}\left(L_{\odot}\right)$ & $4.1 \times 10^{4}$ & $1.0 \times 10^{4}$ & 4 \\
\hline$M_{25-60}\left(M_{\odot}\right)$ & 33 & 9 & 4 \\
\hline$M_{25-100}\left(M_{\odot}\right)$ & 84 & 43 & 4 \\
\hline
\end{tabular}

Note. - Flux densities are denoted by an $S . T_{\mathrm{D}}, L$, and $M$ are dust temperature, bolometric luminosity, and total mass determined from the 25 and $60 \mu \mathrm{m}$ IRAS data when thus indexes, or from the 25, 60, and 100 $\mu$ m data. (1) Becker et al.(1994) (2) Liszt (1992) (3) SIMBAD, a : denotes 
an uncertain value and an L an upper limit. (4) Lis 2006, pers. comm. 
Table 2. Observed Spectral Lines

\begin{tabular}{lccc}
\hline \hline Species & Transition & $\begin{array}{c}\text { Frequency }^{\mathrm{a}} \\
(\mathrm{MHz})\end{array}$ & $\begin{array}{c}E_{1}^{\mathrm{b}} \\
(\mathrm{K})\end{array}$ \\
\hline $\mathrm{CS}$ & $2-1$ & 97980.95 & 2.4 \\
$\mathrm{SiO}$ & $2-1$ & 86848.96 & 2.1 \\
$\mathrm{CH}{ }_{3} \mathrm{OH}$ & $5_{-1}-4_{0} E$ & 84521.21 & 28.4 \\
& $2_{-1}-1_{-1} E$ & 96739.39 & 0.0 \\
& $2_{0}-1_{0} A^{+}$ & 96741.42 & 2.3 \\
& $2_{0}-1_{0} E$ & 96744.58 & 7.6 \\
& $2_{1}-1_{1} E$ & 96755.51 & 15.5 \\
& $0_{0}-1_{-1} E$ & 108893.94 & 0.0 \\
& & & \\
\hline
\end{tabular}


Table 3. Fiducial Positions

\begin{tabular}{rcccc}
\hline \hline & & & & \\
Nr. & $l_{\text {II }}$ & $b_{\text {II }}$ & $\alpha_{\text {J2000 }}$ & $\delta_{\text {J2000 }}$ \\
\hline & & & & \\
1 & 1.5750 & -0.0183 & $17^{\mathrm{h}} 49^{\mathrm{m}} 23.7$ & $-27^{\circ} 35^{\prime} 53^{\prime \prime}$ \\
2 & 1.5939 & -0.0148 & 174918.6 & -273354 \\
3 & 1.6418 & -0.0641 & 174943.6 & -273352 \\
4 & 1.2829 & -0.0289 & 174831.9 & -274927 \\
5 & 1.3690 & -0.1000 & 174913.9 & -274904 \\
6 & 1.3168 & -0.0650 & 174900.8 & -275055 \\
7 & 1.4110 & +0.0260 & 174851.7 & -274229 \\
8 & 1.8053 & -0.3391 & 174801.6 & -273308 \\
9 & 1.48211 & +0.0264 & 174551.6 & -273822 \\
\hline
\end{tabular}

Note. - The first column gives the numbers of the fiducial positions as used in Fig 5 and Table 4. The remaining columns give galactic coordinates and J2000 equatorial coordinates. 
Table 4. Gaussian Fitting Results

\begin{tabular}{|c|c|c|c|c|c|c|}
\hline Pos. & Species & Transition & $\begin{array}{l}T_{\mathrm{A}}^{*} \\
(\mathrm{~K})\end{array}$ & $\begin{array}{c}\int T_{\mathrm{A}}^{*} d v \\
\left(\mathrm{~K} \mathrm{~km}^{-1}\right)\end{array}$ & $\begin{array}{c}v_{\mathrm{LSR}} \\
\left(\mathrm{km} \mathrm{s}^{-1}\right)\end{array}$ & $\begin{array}{c}\Delta v \\
\left(\mathrm{~km} \mathrm{~s}^{-1}\right)\end{array}$ \\
\hline \multirow[t]{8}{*}{1} & $\mathrm{CH}_{3} \mathrm{OH}$ & $5_{-1}-4_{0} E$ & 0.53 & $4.9(0.3)$ & $56.2(0.2)$ & $8.7(0.6)$ \\
\hline & & $0_{0}-1_{-1} E$ & $<0.57$ & - & - & - \\
\hline & CS & $2-1$ & 0.31 & $4.6(4.1)$ & $48.0(5.5)$ & $13.8(6.4)$ \\
\hline & & & 0.71 & $11.0(4.5)$ & $61.2(2.3)$ & $14.4(3.3)$ \\
\hline & & & 0.28 & $8.6(1.3)$ & $163.2(2.1)$ & $28.5(5.5)$ \\
\hline & $\mathrm{SiO}$ & $2-1$ & 0.23 & $6.9(0.4)$ & $53.4(0.6)$ & $27.4(1.1)$ \\
\hline & & & 0.12 & $1.2(0.3)$ & $60.1(0.6)$ & $9.9(1.4)$ \\
\hline & & & 0.10 & $4.1(0.2)$ & $163.3(1.0)$ & $37.0(2.3)$ \\
\hline \multirow[t]{10}{*}{2} & $\mathrm{CH}_{3} \mathrm{OH}$ & $5_{-1}-4_{0} E$ & 0.20 & $3.1(0.3)$ & $51.6(0.8)$ & $14.1(1.7)$ \\
\hline & & $2_{k}-1_{k}$ & \multicolumn{4}{|c|}{ fitting impossible } \\
\hline & & $2_{k}-1_{k}$ & \multicolumn{4}{|c|}{ fitting impossible } \\
\hline & & $0_{0}-1_{-1} E$ & $<0.57$ & - & - & - \\
\hline & CS & $2-1$ & 0.23 & $1.5(0.9)$ & $43.7(1.1)$ & $5.8(3.2)$ \\
\hline & & & 0.62 & $12.2(1.2)$ & $58.7(0.9)$ & $18.6(2.2)$ \\
\hline & & & 0.46 & $12.4(1.0)$ & $162.3(1.0)$ & $25.1(2.2)$ \\
\hline & $\mathrm{SiO}$ & $2-1$ & 0.17 & $1.4(0.3)$ & $42.5(0.4)$ & $7.7(1.3)$ \\
\hline & & & 0.37 & $10.1(0.5)$ & $60.3(0.6)$ & $26.0(1.5)$ \\
\hline & & & 0.21 & $7.0(0.3)$ & $157.9(0.6)$ & $30.8(1.6)$ \\
\hline \multirow[t]{7}{*}{3} & $\mathrm{CH}_{3} \mathrm{OH}$ & $5_{-1}-4_{0} E$ & 0.44 & $4.2(0.2)$ & $51.9(0.3)$ & $8.9(0.8)$ \\
\hline & & $2_{k}-1_{k}$ & 1.3 & $12.6(0.5)$ & $56.2(0.2)$ & $9^{*}$ \\
\hline & & & 1.4 & $13.0(0.6)$ & $*$ & $9^{*}$ \\
\hline & & & 1.4 & $13.3(0.4)$ & $*$ & $9 *$ \\
\hline & & & 0.08 & $0.7(0.4)$ & $*$ & $9^{*}$ \\
\hline & & $0_{0}-1_{-1} E$ & $<0.57$ & - & - & - \\
\hline & CS & $2-1$ & 0.33 & $13(2)$ & $-2(6)$ & $73(15)$ \\
\hline
\end{tabular}


Table 4-Continued

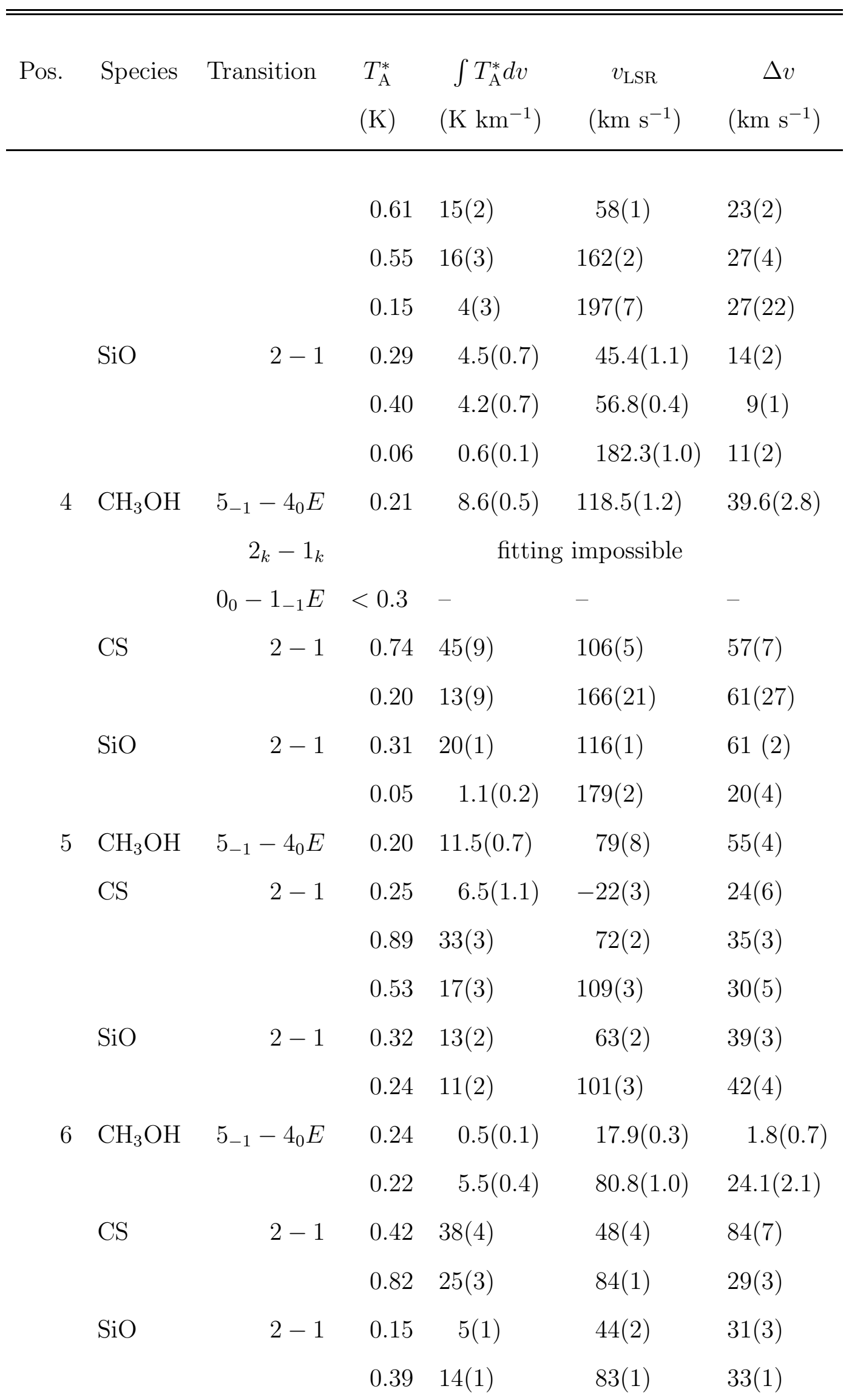


Table 4-Continued

\begin{tabular}{|c|c|c|c|c|c|c|}
\hline Pos. & Species & Transition & $\begin{array}{l}T_{\mathrm{A}}^{*} \\
(\mathrm{~K})\end{array}$ & $\begin{array}{c}\int T_{\mathrm{A}}^{*} d v \\
\left(\mathrm{~K} \mathrm{~km}^{-1}\right)\end{array}$ & $\begin{array}{c}v_{\mathrm{LSR}} \\
\left(\mathrm{km} \mathrm{s}^{-1}\right)\end{array}$ & $\begin{array}{c}\Delta v \\
\left(\mathrm{~km} \mathrm{~s}^{-1}\right)\end{array}$ \\
\hline \multirow[t]{5}{*}{7} & $\mathrm{CH}_{3} \mathrm{OH}$ & $5_{-1}-4_{0} E$ & $<0.2$ & - & - & - \\
\hline & CS & $2-1$ & 0.32 & $9(3)$ & $82(3)$ & $27(5)$ \\
\hline & & & 0.32 & $24(4)$ & $100(5)$ & $69(12)$ \\
\hline & $\mathrm{SiO}$ & $2-1$ & 0.12 & $3.7(0.3)$ & $72(1)$ & $29(2)$ \\
\hline & & & 0.15 & $8.6(0.1)$ & $98(1)$ & $54(2)$ \\
\hline \multirow[t]{3}{*}{8} & $\mathrm{CH}_{3} \mathrm{OH}$ & $5_{-1}-4_{0} E$ & 0.23 & $8.2(0.6)$ & $79.0(1.3)$ & $33.6(3.0)$ \\
\hline & $\mathrm{SiO}$ & $2-1$ & 0.24 & $8(4)$ & $71(1)$ & $32(6)$ \\
\hline & & & 0.16 & $10(4)$ & $85(7)$ & $60(5)$ \\
\hline \multirow[t]{3}{*}{9} & $\mathrm{CH}_{3} \mathrm{OH}$ & $2_{k}-1_{k}$ & \multicolumn{4}{|c|}{ fitting impossible } \\
\hline & $\mathrm{CH}_{3} \mathrm{OH}$ & $5_{-1}-4_{0} E$ & 0.21 & $5(1)$ & $66(1)$ & $24(3)$ \\
\hline & & & 0.12 & $6(1)$ & $99(6)$ & $49(8)$ \\
\hline
\end{tabular}

Note. - Columns are, right to left, position at which spectrum was taken (from Table 3), species, transition, corrected antenna temperature, integrated corrected antenna temperature, LSR velocity, and linewidth (FWHM), with the latter four quantities determined from multi-component Gaussian fits. Meaningful fitting was in most cases impossible for the $2_{k}-1_{k}$ quartet of methanol and modeling of these and other methanol lines are discussed in \$5.1. For the $2_{k}-1_{k}$ fit results for position $4 v_{\mathrm{LSR}}$ and $\Delta v$ are given for the $\sim 55 \mathrm{~km} \mathrm{~s}^{-1}$ system only, with the linewidth fixed to the value of the 
$5_{-1}-4_{0} E$ emission at that velocity. The fitted velocity is that of the $2_{x}-1_{x} A^{+}$ lines and the velocities of the other lines were fixed to it. For that position, the $\mathrm{CH}_{3} \mathrm{OH}$ equivalents of the CS 162 and $197 \mathrm{~km} \mathrm{~s}^{-1}$ components were too difficult to fit. Upper limits given for the $\mathrm{CH}_{3} \mathrm{OH}, \mathrm{O}_{0}-1_{-1} E$ line are 3 times the $1 \sigma \mathrm{rms}$ noise. 
Table 5. $\quad \mathrm{CH}_{3} \mathrm{OH}$ model results: physical parameters

\begin{tabular}{|c|c|c|c|c|}
\hline Pos. & $\begin{array}{l}T_{\mathrm{K}} \\
(\mathrm{K})\end{array}$ & $\begin{array}{l}n\left(\mathrm{H}_{2}\right) \\
\left(\mathrm{cm}^{-3}\right)\end{array}$ & $\begin{array}{c}N\left(C \mathrm{H}_{3} \mathrm{OH}-A\right) \\
\left(\mathrm{cm}^{-2}\right)\end{array}$ & $\begin{array}{c}N\left(\mathrm{CH}_{3} \mathrm{OH}-E\right) \\
\left(\mathrm{cm}^{-2}\right)\end{array}$ \\
\hline \multirow[t]{3}{*}{2} & \multicolumn{4}{|c|}{ high velocity clump } \\
\hline & 190 & $3.6 \times 10^{4}$ & $\begin{array}{l}\quad 5 \times 10^{14} \\
\text { extended cloud }\end{array}$ & $5 \times 10^{14}$ \\
\hline & 30 & $8 \times 10^{4}$ & $9 \times 10^{14}$ & $9 \times 10^{14}$ \\
\hline \multirow[t]{3}{*}{3} & \multicolumn{4}{|c|}{ high velocity clump } \\
\hline & 16 & $4 \times 10^{4}$ & $\begin{array}{l}\quad 1 \times 10^{14} \\
\text { extended cloud }\end{array}$ & $1 \times 10^{14}$ \\
\hline & 60 & $6 \times 10^{4}$ & $6 \times 10^{14}$ & $6 \times 10^{14}$ \\
\hline
\end{tabular}

Note. - Pos. denotes the position (from Table 3) toward which the fitted spectra were taken. $T_{\mathrm{K}}$ and $n\left(\mathrm{H}_{2}\right)$ are the best fit values for the kinetic temperature and the molecular hydrogen density, respectively. $N\left(C \mathrm{H}_{3} \mathrm{OH}-A\right)$ and $N\left(C \mathrm{H}_{3} \mathrm{OH}-\mathrm{E}\right)$ are the best fit column densities of $A$ - and $E$-type methanol, respectively. The calculations assume that both the high velocity clump and the extended cloud are extended relative to the telescope beam. 
Table 6. $\quad \mathrm{CH}_{3} \mathrm{OH}$ model results towards Position 1

\begin{tabular}{|c|c|c|c|c|c|c|}
\hline Transition & $\begin{array}{c}\text { Frequency } \\
\text { (GHz) }\end{array}$ & $\begin{array}{l}T_{o b s} \\
(\mathrm{~K})\end{array}$ & $\begin{array}{c}T_{\text {mod }} \\
(\mathrm{K})\end{array}$ & $\begin{array}{l}T_{o b s} \\
(\mathrm{~K})\end{array}$ & $\begin{array}{c}T_{\text {mod }} \\
(\mathrm{K})\end{array}$ & References \\
\hline & & \multicolumn{2}{|c|}{ extended cloud } & \multicolumn{2}{|c|}{ high velocity clump } & \\
\hline $2_{0}-3_{-1} E$ & 12.179 & -1.25 & -0.25 & -0.38 & -0.24 & 1 \\
\hline $4_{-1}-3_{0} E$ & 36.169 & $\sim 0.4$ & 0.5 & $\sim 0.4$ & 0.26 & 2 \\
\hline $5_{-1}-4_{0} E$ & 84.521 & 0.34 & 0.23 & 0.26 & 0.10 & 3 \\
\hline $2_{-1} \rightarrow 1_{-1} E$ & 96.739 & & & & & \\
\hline $2_{0} \rightarrow 1_{0} A$ & $96.741^{a}$ & 1.85 & 1.63 & 1.05 & 0.66 & 3 \\
\hline $2_{0} \rightarrow 1_{0} E$ & 96.745 & & & & & \\
\hline $0_{0}-1_{-1} E$ & 108.894 & not detected ${ }^{b}$ & 0.2 & not detected ${ }^{b}$ & 0.08 & 3 \\
\hline $8_{0}-8_{-1} E$ & 156.489 & 0.00 & 0 & 0.00 & 0 & 2 \\
\hline $2_{1}-3_{0} A$ & 156.602 & -0.17 & -0.08 & -0.13 & -0.05 & 2 \\
\hline $7_{0}-7_{-1} E$ & 156.829 & 0.00 & 0 & 0.00 & 0 & 2 \\
\hline $6_{0}-6_{-1} E$ & 157.049 & 0.00 & 0 & 0.01 & -0.003 & 2 \\
\hline $5_{0}-5_{-1} E$ & 157.179 & 0.00 & 0 & 0.00 & -0.005 & 2 \\
\hline $4_{0}-4_{-1} E$ & 157.246 & 0.00 & 0 & 0.00 & -0.01 & 2 \\
\hline $1_{0}-1_{-1} E$ & 157.271 & & & & & \\
\hline $3_{0}-3_{-1} E$ & $157.272^{c}$ & 0.09 & 0.2 & 0.17 & 0.11 & 2 \\
\hline $2_{0}-2_{-1} E$ & 157.276 & & & & & \\
\hline
\end{tabular}


Note. - $T_{o b s}$ and $T_{m o d}$ are the observed and the modeled antenna temperatures, respectively. ${ }^{a}$ Blend of $2_{k}-1_{k}, k=0, \pm 1-A, E$ lines. ${ }^{b}$ with an rms of $0.2 \mathrm{~K}$. ${ }^{c}$ Blend of $1_{0}-1_{-1} E, 3_{0}-3_{-1} E$ and $2_{0}-2_{-1} E$ lines. (1) Whiteoak \& Peng (1989); (2) Salii et al. (2002); (3) this work. 


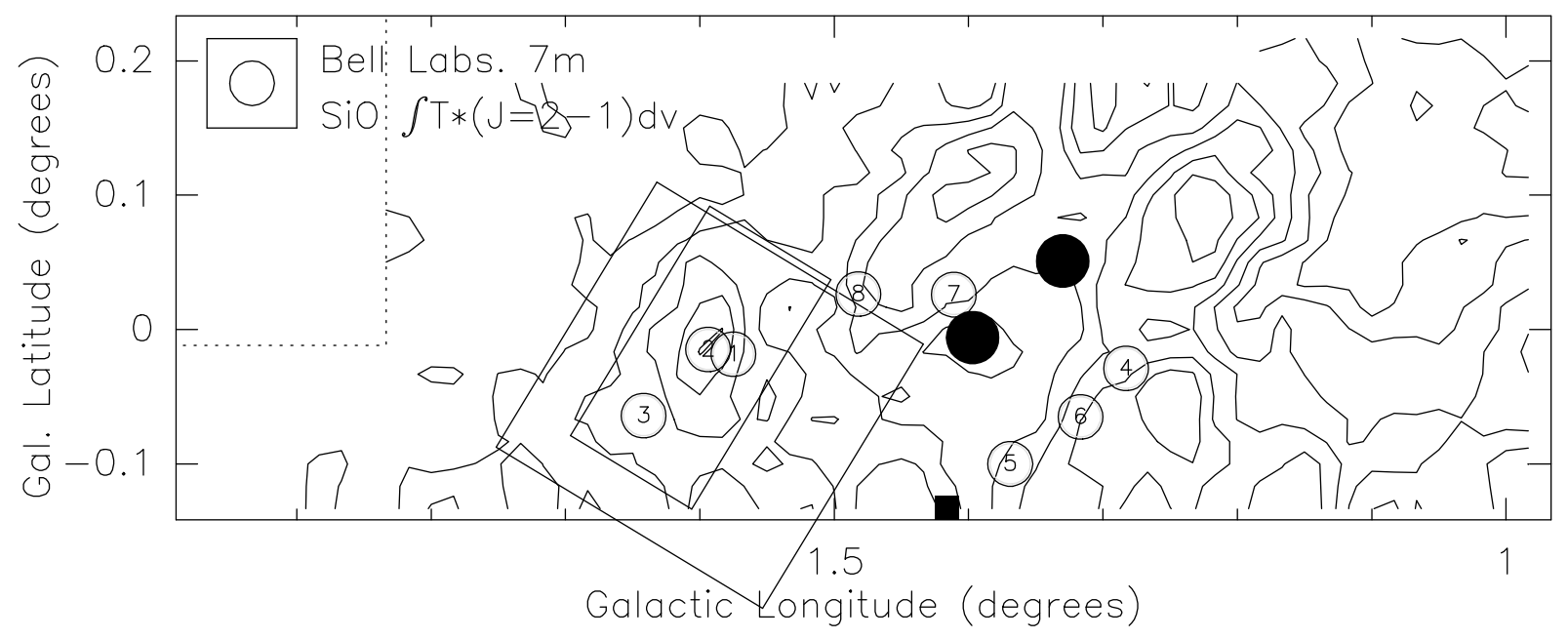

Fig. 1. - Map of integrated $\mathrm{SiO} J=2-1$ emission of G1.6-0.025 made with the Bell Labs. $7 \mathrm{~m}$ telescope. Contours are 2 to 12 in steps of 2 times $1.8 \mathrm{~K} \mathrm{~km} \mathrm{~s}^{-1}$ which is equal to the rms noise. The area within the dotted line was not mapped. The $2^{\prime}$ diameter beam (FWHM) is indicated in the left upper corner of the left upper panel. Fiducial positions lying within the map boundaries are indicated. The square gives the extent of the ammonia map shown in Fig. 3 of Gardner et al. 1985 and the rectangle within it the area mapped by Salii et al. 2002 in methanol lines. Methanol absorption in the $2_{0}-3_{-1} E$ line was mapped by Whiteoak \& Peng 1989 over a roughly similar area as ammonia. The $\mathrm{C}_{3} \mathrm{H}_{2}$ spectra presented by Kuiper et al. 1993 were taken toward various locations within that area. The two dots mark the positions of the radio/FIR sources Sgr D7 (western) and D8 (eastern source). The square marks the position of a $1720 \mathrm{MHz} O H$ maser. 

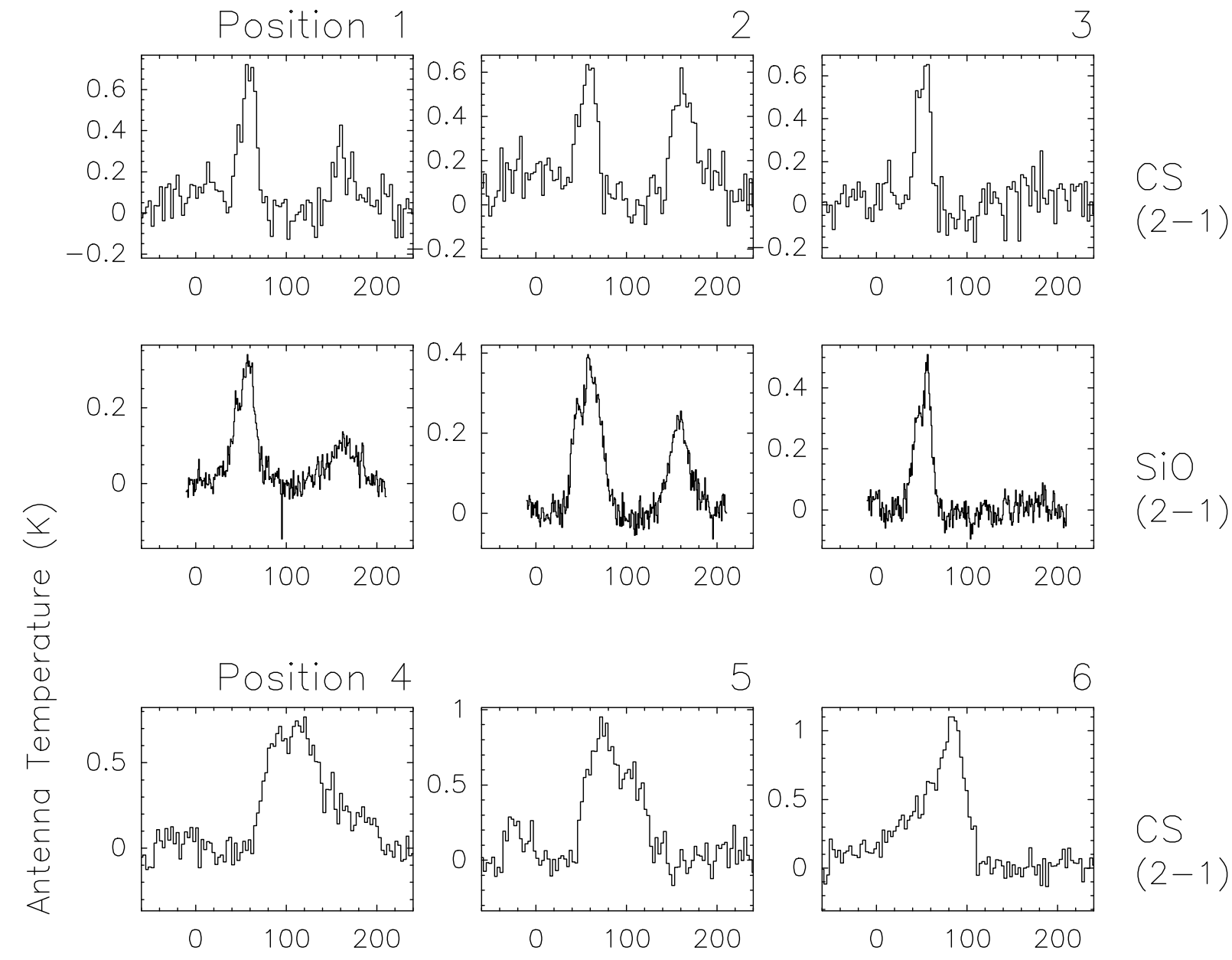

$\mathrm{SiO}$

$(2-1)$
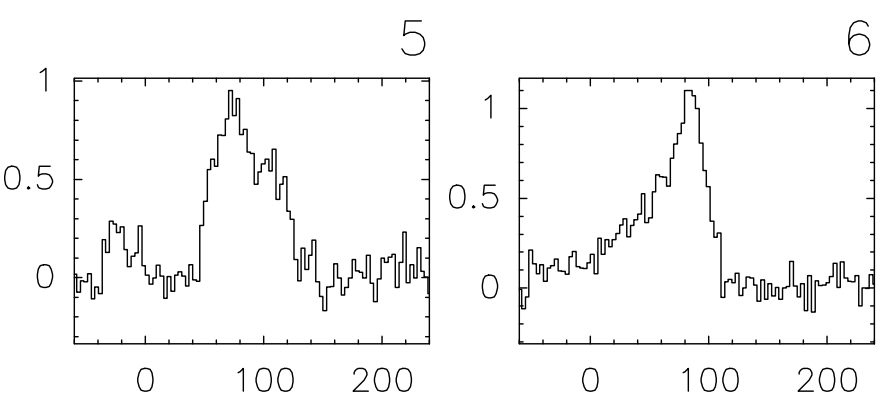

CS

$(2-1)$
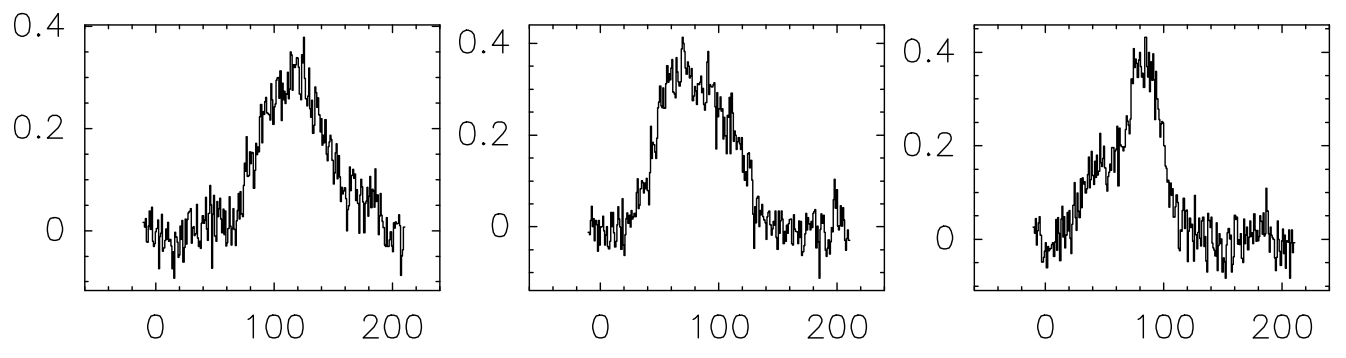

$\mathrm{SiO}$

$(2-1)$

LSR Velocity $\left(\mathrm{km} \mathrm{s}^{-1}\right)$

Fig. 2.- Top to bottom: Spectra taken toward positions 1-6 of Table 3 in the $J=2-1$ lines of $\mathrm{CS}$ and $\mathrm{SiO}$. 


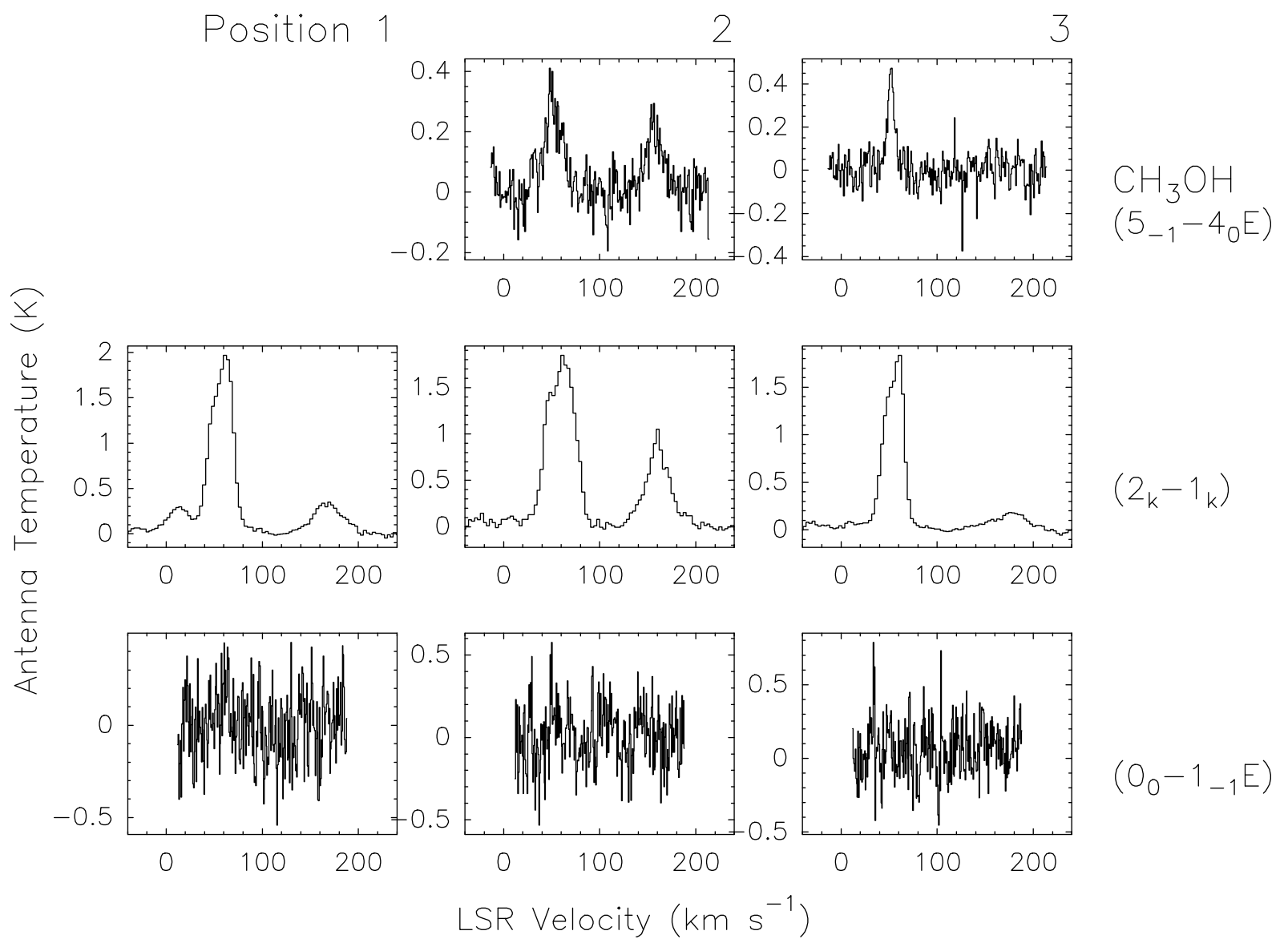

Fig. 3.- Top to bottom: $\mathrm{CH}_{3} \mathrm{OH}$ spectra taken toward positions $1-3$ of the $84 \mathrm{GHz}$ $5_{-1}-4_{0} E$ line, the $2_{k}-1_{k}$ quartet near $97 \mathrm{GHz}$, and the $109 \mathrm{GHz} 0_{0}-1_{-1} E$ line. The $97 \mathrm{GHz}$ quartet is a blend of three $E$-type and one $A^{+}$line (see Table 22) and the LSR velocity scale is relative to the latter. 


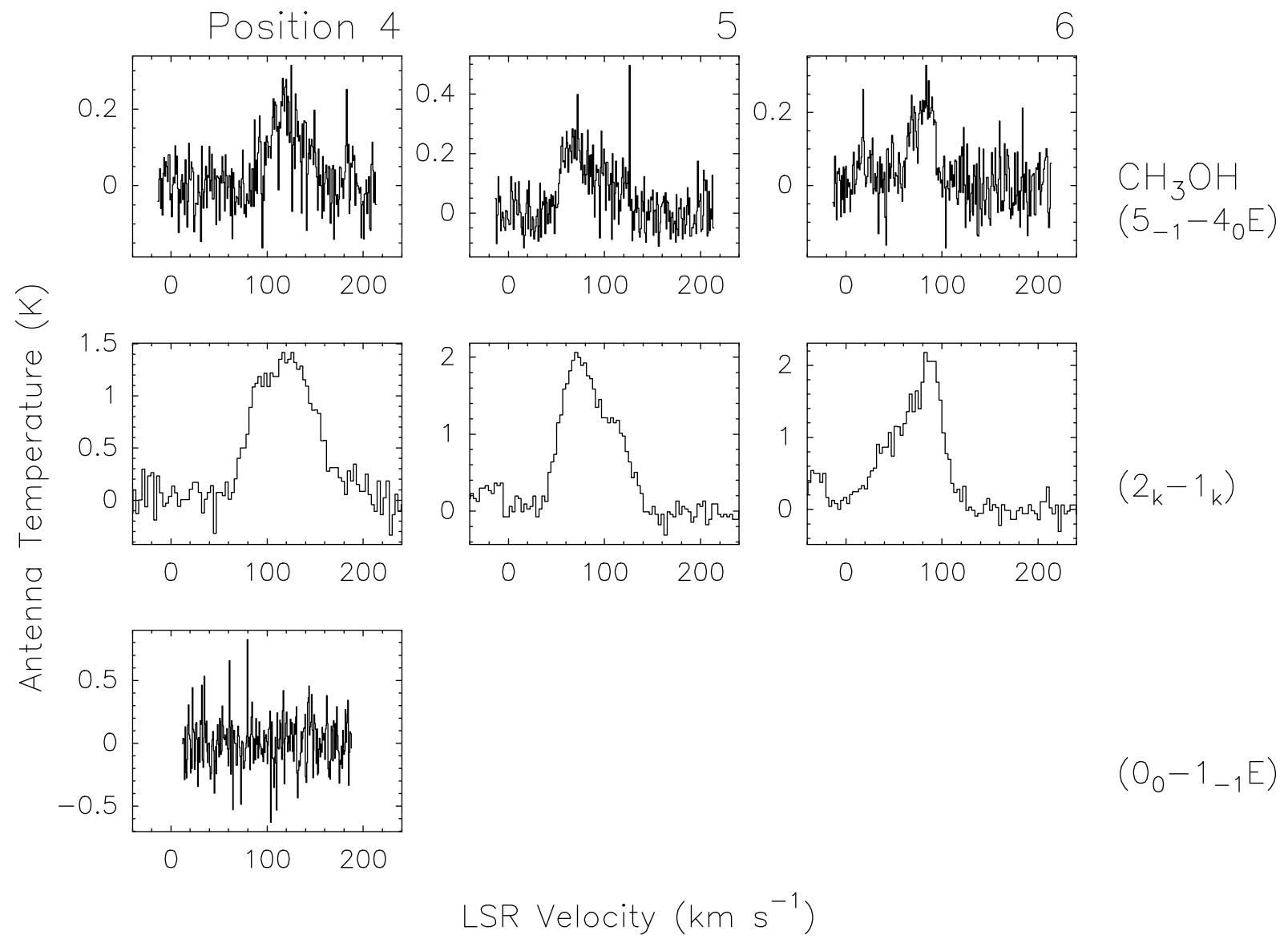

Fig. 4.- Same as Fig. 3 for positions 4-6. 


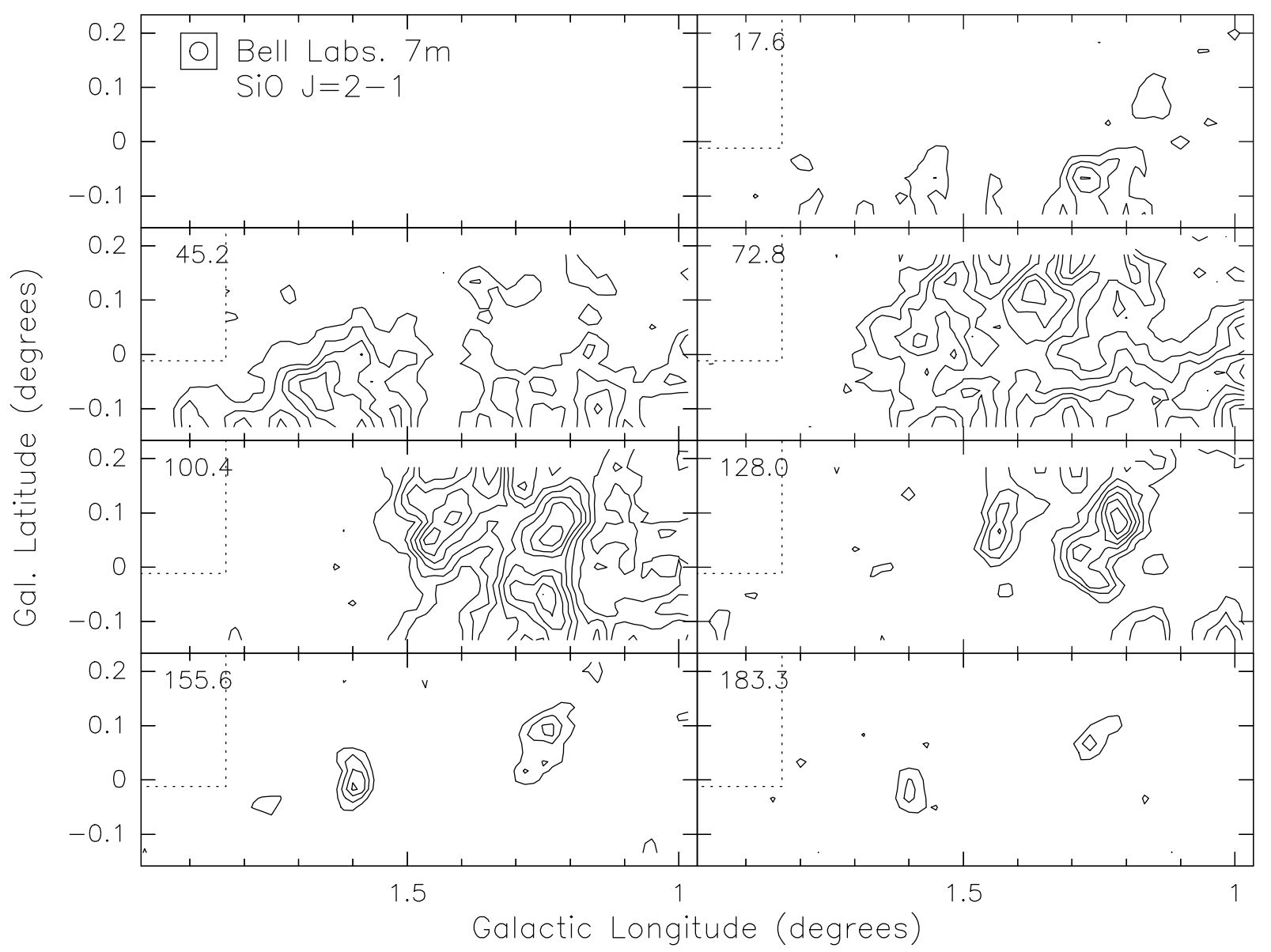

Fig. 5.- Maps of $\mathrm{SiO} J=2-1$ emission of G1.6-0.025 made with the Bell Labs. $7 \mathrm{~m}$ telescope. Each map represents the emission in $T_{\mathrm{A}}^{*}$ units in a "channel" smoothed to a width of $27.6 \mathrm{~km} \mathrm{~s}^{-1}$ centered on the velocity (in $\mathrm{km} \mathrm{s}^{-1}$ ) given in the left upper corner of each panel. Contours are 2 to 20 in steps of 2 times $25 \mathrm{mK}$, which is equal to the rms noise. The area within the dotted lines was not mapped. An additional area of width $-0.11<b<0.0$ was also mapped from $l=2$. to 2.6 but no emission was found within it. The $2^{\prime}$ diameter beam (FWHM) is indicated in the left upper corner of the left upper panel. 

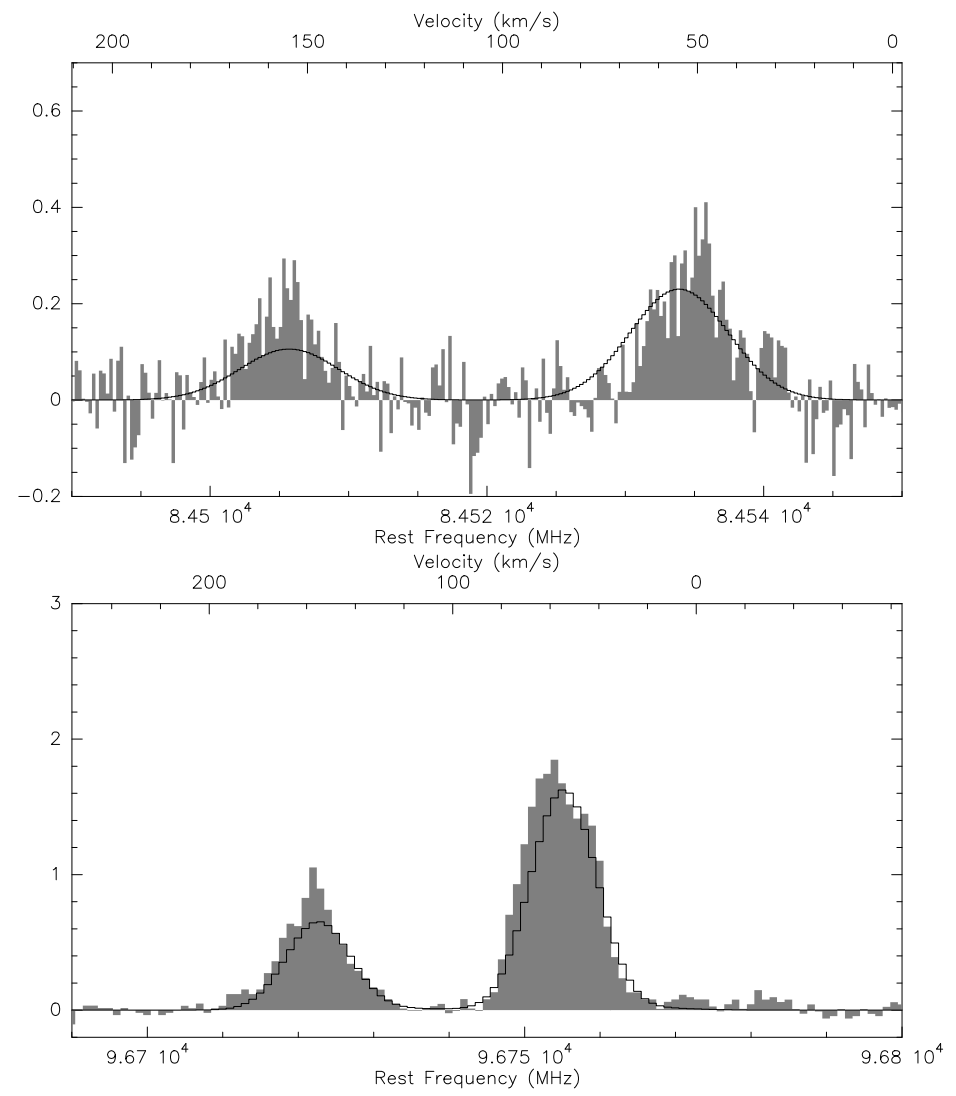

Fig. 6.- Best LVG fit toward position 2 overlaid on the real data for the $5_{-1}-4_{0} E$ line upper panel and the $2_{k}-1_{k}$ quartet of lines lower panel. 

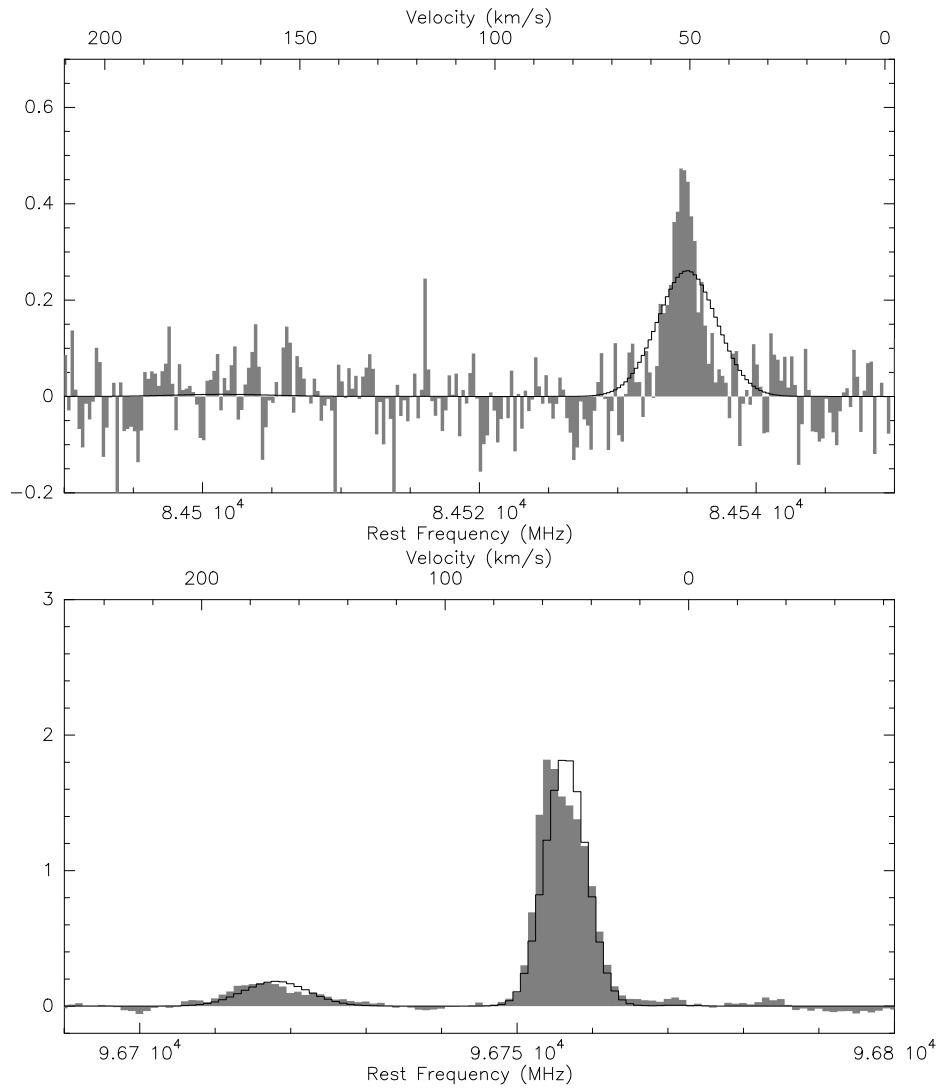

Fig. 7.- Same as Fig. 6 for position 3. 


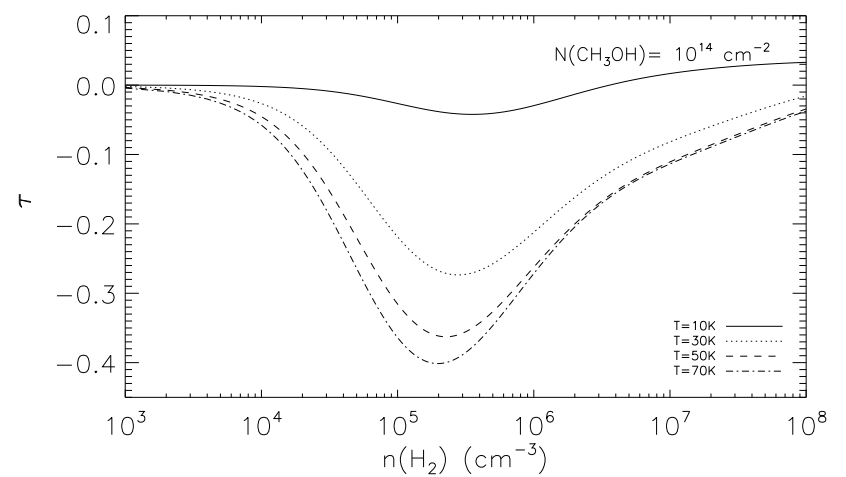

(a)

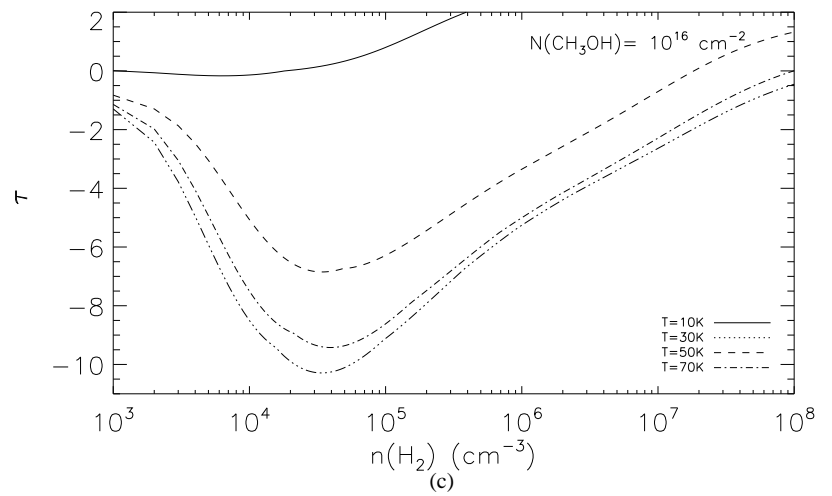

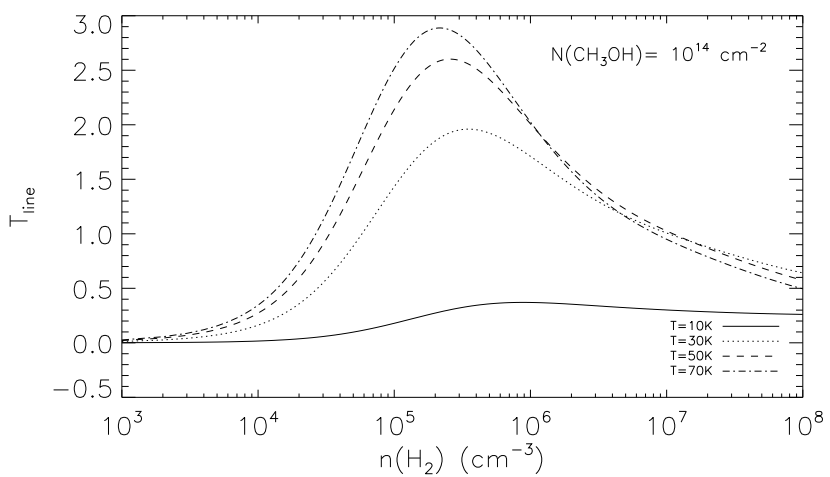

(b)

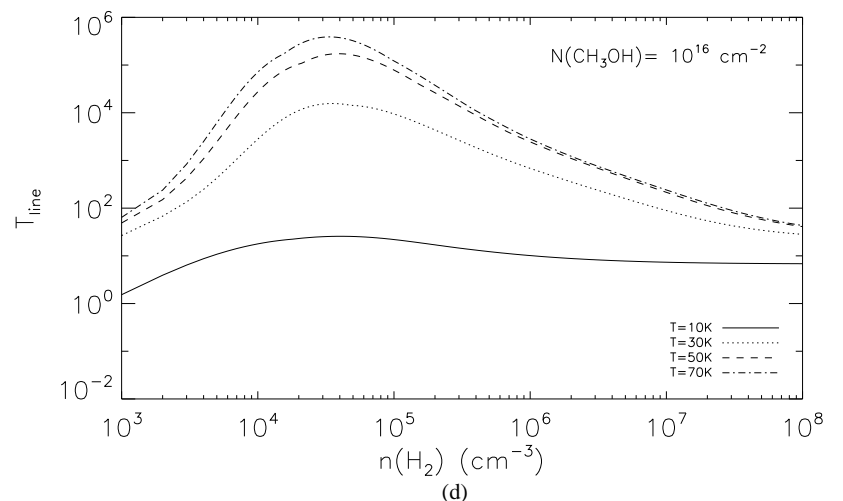

Fig. 8. - Line optical depths (left panels) and line intensities (right panels) of $5_{-1}-4_{0} E$ line as function of different temperatures, for two $\mathrm{CH}_{3} \mathrm{OH}-A$ column densities, $10^{14} \mathrm{~cm}^{-2}$ (upper panels) and $10^{16} \mathrm{~cm}^{-2}$ (lower panels). 

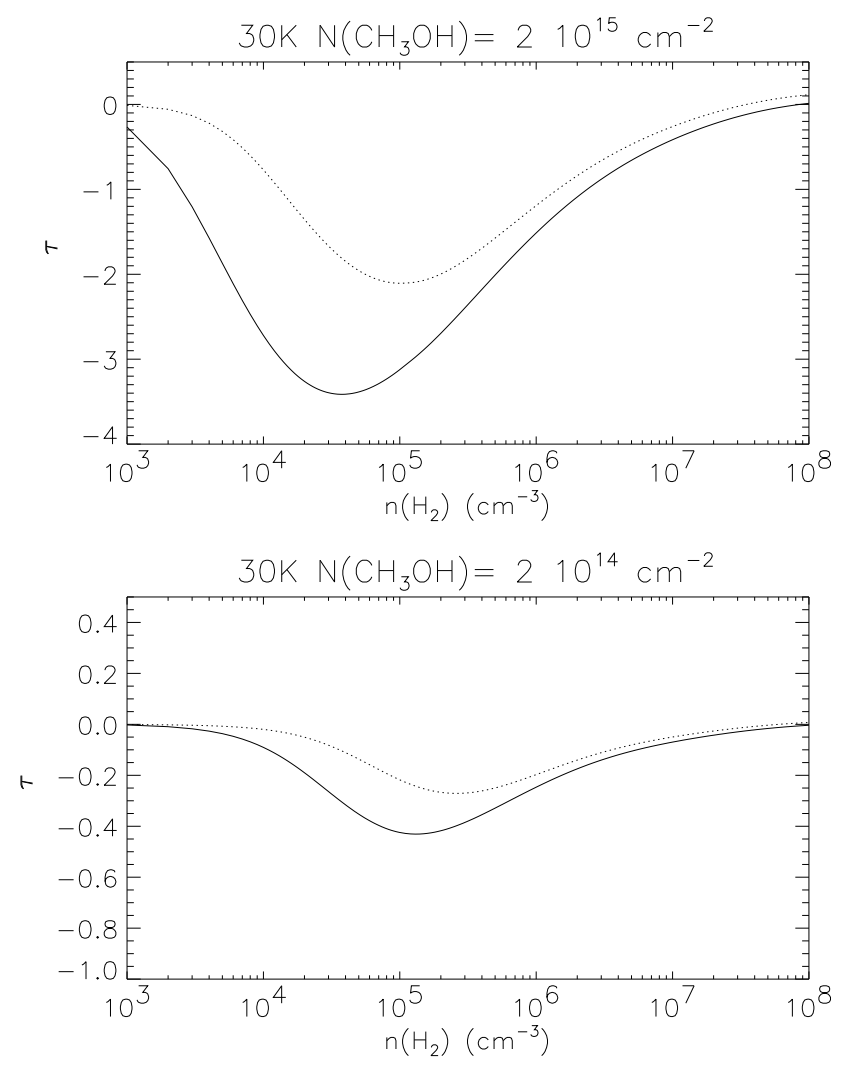

Fig. 9.- Line optical depths of $4_{-1}-3_{0} E$ (solid line) and $5_{-1}-4_{0} E$ (dotted line) as function of density at different $\mathrm{CH}_{3} \mathrm{OH}$ column densities, $210^{15} \mathrm{~cm}^{-2}$ upper panel and $210^{14} \mathrm{~cm}^{-2}$ lower panel. 